\title{
A Search for HAeBe stars in the bar of the Large Magellanic Cloud based on optical variability
}

\author{
W. J. de Wit ${ }^{1,3}$, J. P. Beaulieu ${ }^{2}$, and H. J. G. L. M. Lamers ${ }^{1,3}$ \\ 1 Astronomical Institute, University of Utrecht, Princetonplein 5, 3584CC, Utrecht, The Netherlands \\ 2 Institut d'Astrophysique de Paris, 98bis Boulevard Arago, 75014 Paris, France \\ 3 SRON Laboratory for Space Research, Sorbonnelaan 2, NL-3584 CA, Utrecht, The Netherlands
}

Received 29 June 2000 / Accepted 30 May 2002

\begin{abstract}
We searched the EROS1 database consisting of 80000 stars in the bar of the Large Magellanic Cloud for blue objects with irregular photometric behavior similar to Galactic Herbig Ae/Be (HAeBe) stars. We found 14 new objects in addition to the discovery of $7 \mathrm{HAeBe}$ candidate stars found in our previous studies. Based on their light curve and colours we classify these new objects also as EROS LMC HAeBe Candidates (ELHCs). The objects are located in an area of enhanced $60 \mu \mathrm{m}$ emission, which indicates a star forming region. The stars are concentrated in three areas and the majority of the objects are clustered round the N 120 nebular complex. The light curves are interpreted with a simple model, involving obscuration by an inhomogeneous orbiting circumstellar dust cloud as the dominant cause of the variability. We find that the dust orbiting the stars is at a radial distance in the order of 10 to a few 100 AUs. Most of the objects are "bluer-when-fainter". This is attributed to the presence of a (blue) scattering nebula with a size smaller than the point spread function of the photometric images. The stellar parameters are derived from the photometry. The luminosity of the stars is in the range of $3.4<\log \left(L / L_{\odot}\right)<4.2$ and the colours indicate spectral types $\mathrm{O}$ or $\mathrm{B}$. The stars are located in the HR-diagram above the birthline for Galactic stars by about $1 \mathrm{dex}$ in luminosity. The method used to detect the ELHCs stars on optical variability criteria is discussed. We show that our sample of HAeBe candidates in the EROS 1 field of view is complete up to $V \simeq 16.5^{\mathrm{m}}$ for the typical amplitude and time scale of the ELHCs.
\end{abstract}

Key words. stars: formation - stars: pre-main sequence - stars: variables: general - reflection nebulae - Magellanic Clouds methods: data analysis

\section{Introduction}

The effect of metallicity on star formation and early stellar evolution is not known, though it is for instance of importance for the interpretation of the integrated characteristics of high redshift galaxies. The properties of the recently discovered intermediate mass Pre-Main Sequence (PMS) candidate stars (Beaulieu et al. 1996; Lamers et al. 1999) in the Large Magellanic Cloud (LMC) may contribute to the understanding of star formation in environments with different metallicity than the Galaxy. In Lamers et al. (1999) (henceforth refered to as LBD) it is shown that the optical properties of these stars are very similar to the ones of Galactic intermediate mass PMS stars. However, one significant difference was found: the LMC Pre-Main Sequence candidate stars seem systematically more luminous than their Galactic counterparts. The luminous nature of the LMC objects may indicate a higher proto-stellar mass accretion rate at lower metallicity.

Send offprint requests to: W. J. M. de Wit, e-mail: dewit@arcetri.astro.it
Intermediate mass ( 2 to $10 M_{\odot}$ ) PMS stars were grouped for the first time by Herbig (1960) as A or B type stars with emission line spectra and which are associated with nebulosities. Herbig conjectures them to be the higher mass analogues of the TTauri stars. The stars are called Herbig Ae/Be stars, HAeBes or plainly Herbig stars. HAeBes have gained much interest during the last two decades as they provide clues to the poorly understood processes governing star formation in the intermediate mass range. As a result the total number of known HAeBes has grown since. Reviews are given by Thé (1994) and Waters \& Waelkens (1998), where the latter authors propose the following working definition for HAeBe stars:

(1) spectral types A or B with emission line spectra;

(2) luminosity class III to V;

(3) circumstellar infrared excess emission.

A commonly observed property of HAeBe stars (and PMS stars in general) is irregular brightness variability. Herbst \& Shevchenko (1999) show on the basis of photometric monitoring of about 230 stars since 1983, that virtually all HAeBe stars are variable. 
The first LMC HAeBe candidate stars were selected by Beaulieu et al. (1996) on the basis of their colours (A or B type stars) and their peculiar optical brightness variability, which resembles most closely the type of variability observed among Galactic HAeBe stars. The more elaborate optical study presented by LBD confirmed a B spectral type for the majority of the ELHCs and the presence of strong $\mathrm{H} \alpha$ emission. They suggested the name ELHC or EROS LMC HAeBe Candidates, which we use throughout this paper.

The brightness variability of Galactic HAeBe stars is generally explained in terms of extinction and scattering properties of a non-homogeneous gas/dust distribution in their circumstellar (CS) environment, as first proposed by Wenzel (1969). The observed variability properties of Galactic HAeBe stars show that stars later than spectral type B8 can display a much larger amplitude (Finkenzeller \& Mundt 1984; Van den Ancker et al. 1998; Herbst \& Shevchenko 1999). The amplitude can extend up to $\Delta V \approx 3^{\mathrm{m}}$ or larger. This collection of large amplitude PMS variables was suggested to have the name UXors (Herbst et al. 1994), after their prototype UX Orionis. Typical for UXors is their outstanding colour variability. The observed colour, gradually getting redder as the star fades, reverses at some point this trend and starts to become bluer (Wenzel 1969; Zaitseva 1987; Natta \& Whitney 2000). This phenomenon is called the blueing effect and is only observed when the UXor fades into a deep brightness minimum. It is explained in terms of the increasing relative contribution of blue scattered radiation when the star gets obscured by a dust cloud (see Grinin 1988).

In LBD it is found that five of the seven ELHCs display a bluer colour when the star faints. Combined with their peculiar irregular brightness changes, it is suggested that the ELHCs are subject to a similar variability mechanism as the PMS UXor subgroup. However the blueing effect of ELHCs is explained somewhat differently from the one observed among the UXors. For the ELHCs, the blue scattered radiation originates in an extended circumstellar (CS) nebula, which is unresolved at LMC distance (see LBD, for a more elaborate discussion). The ELHC nebulae might be similar to the reflection nebulae of the A and B type stars, which Herbig (1960) selected to compile the first HAeBe catalogue. In contrast it is thought that the scattered radiation of UXors originates in the direct circumstellar vicinity (few AUs) of the star, i.e. most likely scattered off a CS accretion disk.

Currently, seven ELHCs have been discovered and discussed. The objective of this paper is to investigate the properties of ELHCs systematically. Therefore we search the EROS1 data base for objects in the LMC bar with similar photometric variability characteristics as the initial sample of seven ELHCs.

In Sect. 2 we describe the observations and in Sect. 3 we give the results of our search for HAeBe candidates. Section 4 describes the location of the ELHCs in the bar of the LMC. In Sect. 5 we derive the properties of the ELHCs and their location in the HR-diagram. The light curves are characterized and interpreted in Sect. 6. Section 7 treats quantitatively the difference between used variability search methods, viz. Analysis of Variance and the Modified Periodogram Method. Section 8 deals with the number of ELHCs found with the AoV search method. Finally, the results are discussed in Sect. 9.

\section{Observations: The EROS1 catalogue}

The photometric observations are the product of the EROS1 microlensing survey of their 1991-1992 campaign. See Beaulieu et al. (1995), Grison et al. (1995), and Sect. 2 of LBD for a detailed presentation of the data set. For a full description of the EROS1 equipment see Arnaud et al. (1994a, 1994b). To summarize, this data set is made of 2500 images of an area of $1.0 \times 0.4$ degrees, centred on the bar of the LMC. The observations span a period of over 120 days and were taken in two broad band filters, $B_{\mathrm{E}}$ and $R_{\mathrm{E}}$. The $B_{\mathrm{E}}$ passband is close to that of Johnson $V$ band, but it is broader. The $R_{\mathrm{E}}$ passband is intermediate between Cousins $R_{\mathrm{C}}$ and $I_{\mathrm{C}}$. The magnitude scale is defined such that the intrinsic colour of an A0 star has $\left(B_{\mathrm{E}}-R_{\mathrm{E}}\right)=0$, while the zero points are such that a star of zero colour will have a value for its $B_{\mathrm{E}}$ magnitude that will equal its Johnson $B$ magnitude. Conversion equations from the EROS1 to the Johnson system is reported in LBD. Light curves of about 80000 stars with an excellent phase coverage ( $\sim 10$ measurements per night for 120 nights) have been built.

\section{HAeBe candidates in the EROS1 catalogue}

In this section we will present the search method and selection of blue irregular variable stars in the EROS1 light curve database. Results on Cepheid variables (Beaulieu et al. 1995, 1997a, 1997b; Sasselov et al. 1997) and Eclipsing Binaries (Grison et al. 1995) in this database have been presented in the literature.

\subsection{Selection of variable stars}

We conducted a systematic search for photometric variability and identified variable stars using the one way Analysis of Variance (AoV) method of Schwarzenberg-Czerny (1989). This method differs from the one used by LBD. In LBD the Modified Periodogram (MPG) method (Grison 1994) was used to search for variability. The MPG method, based on Fourier harmonics, is very sensitive to periodic signals. It is expected that a large power will be generated at the appropriate frequencies, as long as a limited amount of Fourier harmonics are required to build the signal. On the other hand, the AoV method may be more sensitive to quasi-periodic or a-periodic signals, because it is based on trial periods and the phase-binning principle. We will elaborate on the difference between AoV and MPG in Sect. 7.

Before subjecting all 80000 light curves of the EROS1 database to the AoV method, unreliable data points and spurious measurements are removed. A first selection for stars with large amplitude of variation is made using a $\chi^{2}$ statistic. This results in 15551 objects. We search among these objects for variable stars using the AoV method.

The EROS1 photometry has an observing window of $\sim 120$ days, and we construct AoV periodograms for trial periods between 0.5 and 100 days. The power threshold level for 


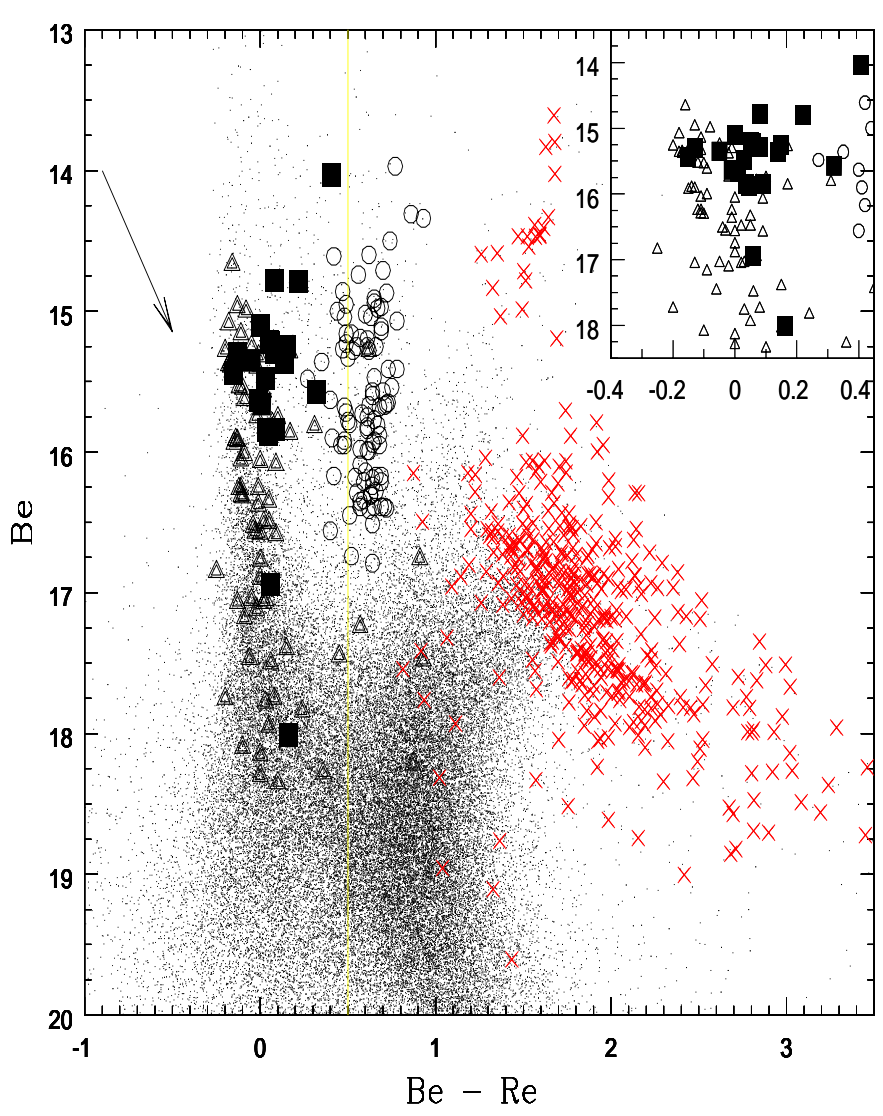

Fig. 1. Observed colour-magnitude diagram of the approximately 80000 stars within the EROS1 catalogue, centred on the bar of the LMC. Indicated are the Cepheids from Beaulieu et al. (1995) in open circles, the Eclipsing Binaries from Grison et al. (1995) in open triangles and the red long term pulsating (Miras) and red irregular variables in crosses. The filled squares are the HAeBe candidates. The arrow indicates the reddening vector in the EROS1 photometry for an $E\left(B_{\mathrm{E}}-R_{\mathrm{E}}\right)=0.5$ according to Sasselov et al. (1997). The vertical line corresponds to the adopted colour criterion used in identifying the HAeBe candidates. Inset: a blow-up of the region in the CMD where the HAeBe candidates are found.

detection of variability is fixed empirically at 30 after inspections of a large number of light curves. The EROS1 noise is found to have non-Gaussian components, the characteristics of which is easily identifiable by inspecting the large number of light curves available. We estimated the typical value for the noise power at $90 \%$ confidence level to be of 20 for a star of $19^{\mathrm{m}}$. This finally resulted in a sample of 1058 variable stars, both regular and irregular.

\subsection{Searching for HAeBe stars}

$\mathrm{HAeBe}$ stars are predominantly blue stars and display an irregular variability with a large range of time scales and amplitudes (e.g. Bibo \& Thé 1991; Herbst \& Shevchenko 1999). Therefore, we select candidate HAeBe stars on the basis of these two criteria, like in Beaulieu et al. (1996) and LBD. First we exclude stars with $\left(B_{\mathrm{E}}-R_{\mathrm{E}}\right)>0.5$, satisfying the selection constraint for HAeBes to be B or A type. For this colour criterion we take into account the known extinction towards the LMC as well as the extinction in the bar of the LMC. The colour limit then corresponds to selecting Main Sequence stars with a spectral type A9V or earlier. The colour limit excludes the majority of Post-Main Sequence variable stars like Cepheids and Long Period Variables as well. We cross identify every selected variable star with published EROS1 Eclipsing Binaries and Cepheid catalogues. Finally, we subject the light curves of the remaining stars to two tests. Firstly, we performed a visual inspection of every light curve, which resulted in the identification of twelve Eclipsing Binaries which were not reported in the EROS1 catalogue of Grison et al. (1995). Secondly we performed a cross-correlation analyses of the $R_{\mathrm{E}}$ light curves and $B_{\mathrm{E}}$ light curves to separate noisy light curves from light curves of stars which are intrinsically variable, but irregularly.

The final sample consists of 21 stars. Their light and colour curves are presented in Figs. 2 and 3. These stars form the sample of the EROS1 LMC HAeBe Candidates. ELHC 1 to 7 were described in LBD. Their light curves exhibit similar quasiregular or irregular variability characteristics in both $R_{\mathrm{E}}$ and $B_{\mathrm{E}}$. These variations have a high probability of being intrinsic to the stars themselves. The coordinates of the 21 ELHCs, their EROS1 identification names and the finding charts are presented in the appendix.

\subsection{The colour-magnitude diagram of variable stars}

The colour-magnitude diagram of the approximately 80000 stars within the EROS1 field is presented in Fig. 1. All identified variable stars in the field have been indicated. The ELHCs are presented by filled squares, Eclipsing Binaries by triangles, Cepheids by open circles and the Long Period Variables by crosses. The location of the ELHCs in the colour-magnitude diagram shows that the majority are bright objects with $B_{\mathrm{E}}<16^{\mathrm{m}}$ (adopted LMC distance modulus is $\sim 18.5$, Walker 1999). Only two objects (ELHC 7 and 9) have a lower brightness. The bright nature of the ELHCs is accompanied by blue colours. We see from the inset in Fig. 1 that only three out of twenty-one ELHCs have $\left(B_{\mathrm{E}}-R_{\mathrm{E}}\right)>0.2$. This corresponds to a colour corrected for LMC foreground extinction $(E(B-V)=0.17$, Fitzpatrick 1986) of $(B-V)_{\mathrm{J}} \simeq-0.05$. We conclude that both the colour and brightness of virtually all ELHCs is indicative for a $B$ spectral type or earlier.

\section{The location of ELHCs in the LMC bar: Correlation with infrared emission}

We present in Fig. 4 the location in the LMC of the 21 ELHCs. The two panels show the same $2.0 \times 3.0$ degrees region centred on $(\mathrm{RA}, \mathrm{Dec})=(05: 25: 12,-69: 17: 40)$ in J2000, but observed in two different wavelength bands. The top panel is an IRAS $60 \mu \mathrm{m}$ image, the bottom panel is an optical image retrieved from the Digital Sky Survey. The ELHCs are indicated only in the bottom panel by small filled dots. Contours of the top $60 \mu \mathrm{m}$ image have been drawn over the bottom visual image to facilitate comparison between the two. The large and the small squares in the optical image (bottom panel) represent the areas, where we searched for blue variable stars. 


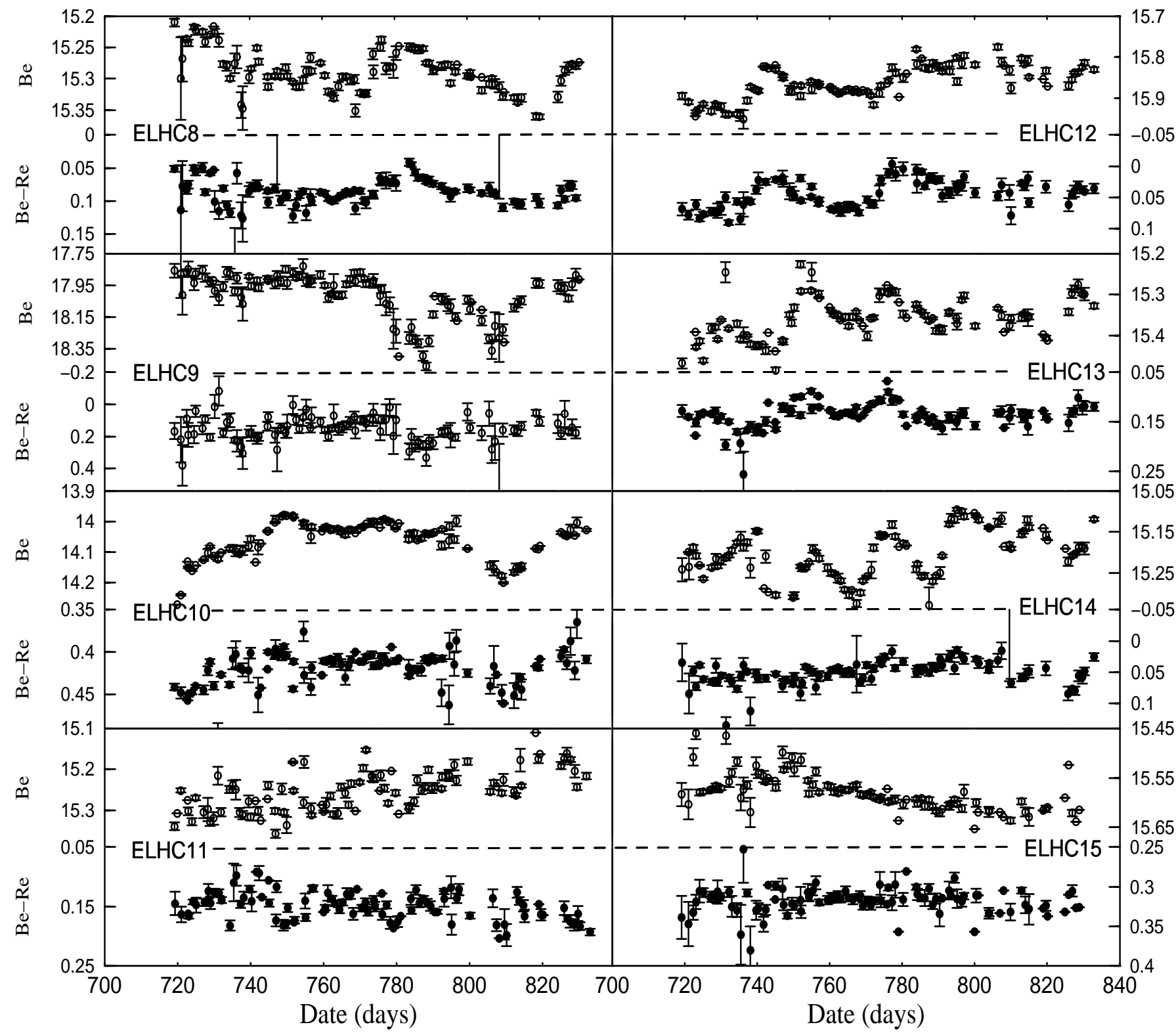

Fig. 2. The light curves in $B_{\mathrm{E}}$ and colour curves in $B_{\mathrm{E}}-R_{\mathrm{E}}$ for the new HAeBe candidates ELHC 8 to ELHC 15 in the Large Magellanic Cloud. Dates are since Jan. 11990 (JD = 2447862.5). Presented points are averaged by day (if there are more than 3 measurements taken on that day).

The $60 \mu \mathrm{m}$ emission traces the presence of dust and can be used as an indicator for massive star formation (Mead et al. 1990). The IRAS $60 \mu \mathrm{m}$ image of the central parts of the LMC shown in the top panel therefore identifies sites of ongoing or recent star formation. Note the strong emission on the east side (east is left and north is up), originating from the massive star forming region 30 Dor. The figure clearly shows that the large majority of the ELHCs (filled dots) are associated with $60 \mu \mathrm{m}$ emission region which traverses the LMC bar from north-west to south-east. In general, the ELHCs lie at or near the edges of the $60 \mu \mathrm{m}$ complex.

The objects are concentrated roughly in three areas with corresponding coordinate offsets in Fig. 4: group 1 near $\left(-30^{\prime}\right.$, $\left.+25^{\prime}\right)$, group 2 near $\left(-45^{\prime},+10^{\prime}\right)$, and group 3 near $\left(+15^{\prime}\right.$, $\left.+30^{\prime}\right)$.
Group 1 is the largest group of stars. They cluster around the nebular complex N 120, which is a complex system which comprises two wind blown bubbles, a supernova remnant and several Hin regions (Laval et al. 1992). One object, viz. ELHC 2, is projected inside this complex.

The objects in group 2 are bound to the north by the N 119 H II region, nearby to the blue globular sized cluster NGC 1903.

Group 3 consists of $3 \mathrm{HAeBe}$ candidates. These objects are projected on top of each other in Fig. 4 and are therefore represented by just one dot. These three candidates do not seem to be associated with $60 \mu \mathrm{m}$ emission.

Finally, the HAeBe candidate ELHC 21 is associated with the supernova remnant SNR 0520-69.4.

We conclude that the location of the ELHCs in the bar of the LMC supports the notion of young objects, and possibly 


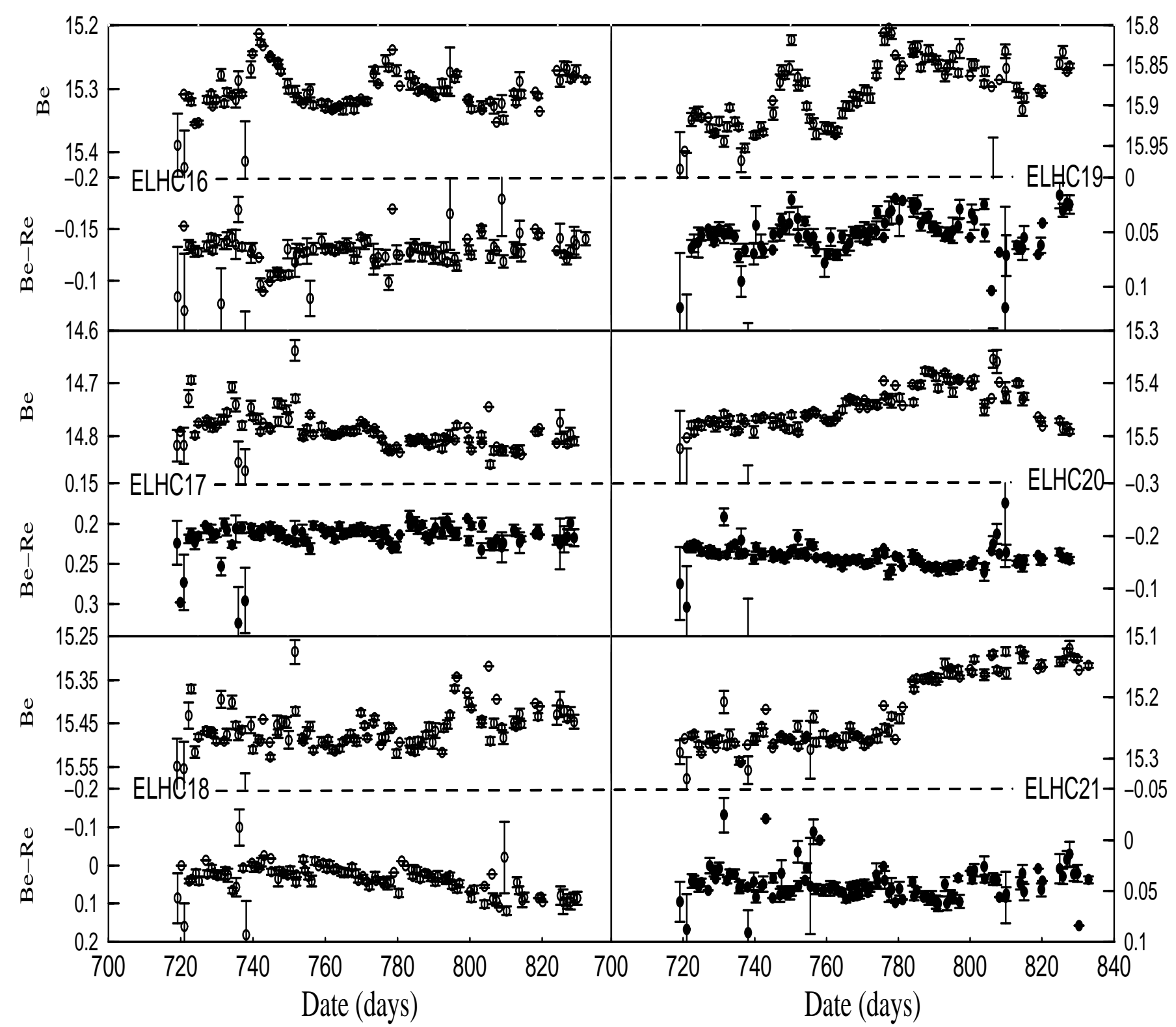

Fig. 3. The same as Fig. 2, but for HAeBe candidates ELHC 16 to ELHC 21.

Pre-Main Sequence. However we cannot exclude the possibility that the found correlation between the location of the ELHCs and the $60 \mu \mathrm{m}$ excess emission is a chance projection.

\section{Properties of the ELHC stars}

In the following subsections we discuss the properties of the ELHC stars derived from their magnitude, colour and type of variability. These properties are tabulated in Table 1.

\subsection{The light curves}

The light curves in the $B_{\mathrm{E}}$ filter band and the colour curves in $\left(B_{\mathrm{E}}-R_{\mathrm{E}}\right)$ of ELHC 8 to 21 are shown in Figs. 2 and 3. The light curves of ELHC 1 to 7 are published by LBD. The figures show the irregular or quasi-regular nature of the brightness variability, which may be best described as "bumpy".
The characteristic time scale of the variation is around twenty days. Some ELHCs show trends on a longer time scale, e.g. ELHC 8, 15 and 20. The variations have amplitudes between $0.15^{\mathrm{m}}$ and $0.50^{\mathrm{m}}$ in $B_{\mathrm{E}}$. The largest amplitude is displayed by the two faintest stars, ELHC $7\left(B_{\mathrm{E}} \simeq 17.0^{\mathrm{m}}\right)$ and ELHC 9 $\left(B_{\mathrm{E}} \simeq 18.0^{\mathrm{m}}\right)$. On the contrary, no large amplitude variations with $\Delta B_{\mathrm{E}} \gtrsim 0.45^{\mathrm{m}}$ exist among the bright majority of the ELHCs.

We classify the observed brightness variations in two ways. The first involves the change of the colour of the object as function of the change in brightness, i.e. the colour gradient $\mathrm{d}\left(B_{\mathrm{E}}-R_{\mathrm{E}}\right) / \mathrm{d} R_{\mathrm{E}}$. The second classification involves the quantification of the time a star spends at a certain brightness level with respect to the average brightness, viz. more often bright than faint or vice versa. The classifications may reveal indications of the mechanism which causes the observed variability. 

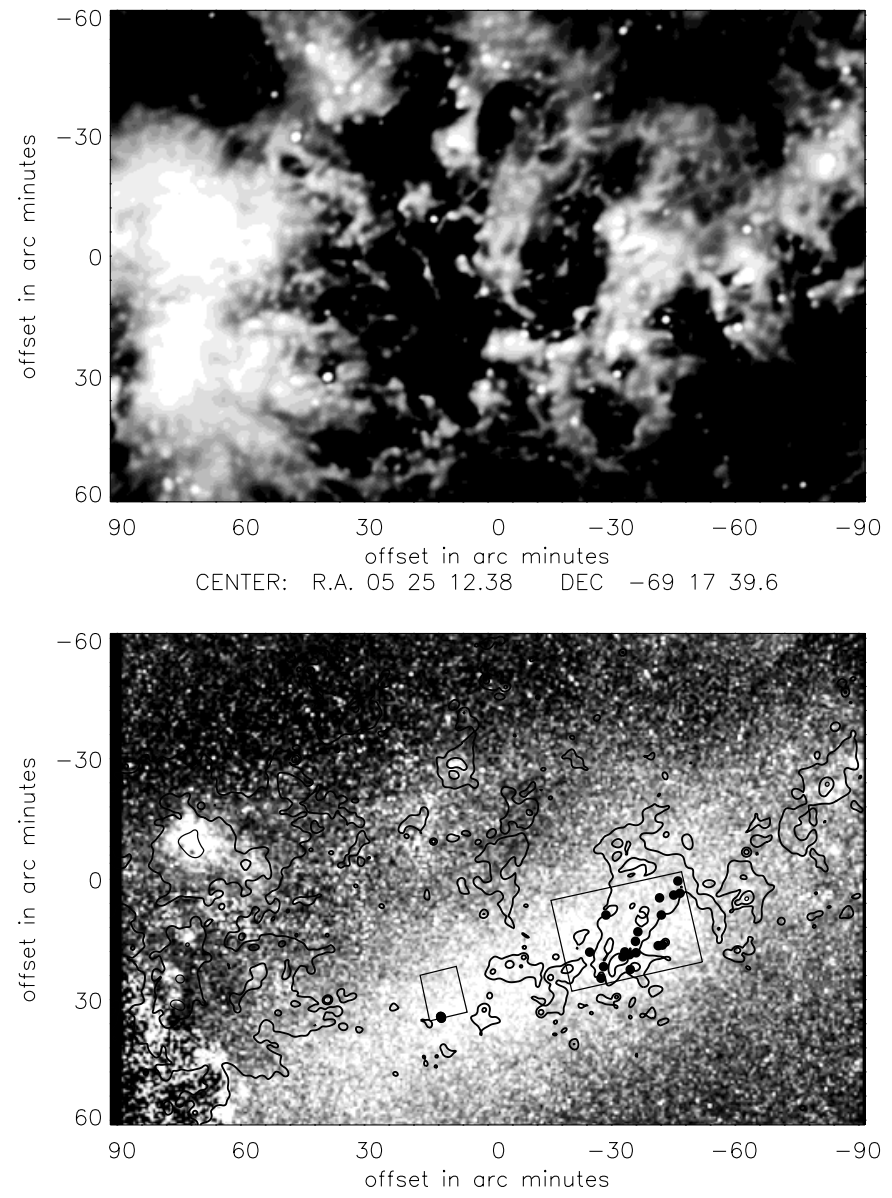

Fig. 4. Top panel: Newly processed IRAS image at $60 \mu \mathrm{m}$ of the central parts of the LMC. North is up, east is left. The large emission region on the left is 30Dor. Bottom panel: Digital Sky Survey image of the same area of the LMC. For comparison we have put $60 \mu \mathrm{m}$ contours at 25, 100 and $1000 \mathrm{MJy} / \mathrm{sr}$ on top. Indicated are the ELHCs (black dots) and the searched areas (squares). Clearly the large majority of the ELHCs are positioned in or near the edge of the $60 \mu \mathrm{m}$ emission region in the bar of the LMC.

\subsubsection{The colour gradient}

The analyses of LBD shows that ELHC 1 to 5, have a brightness variability which is characterized by a distinctive bluer colour during brightness minima, i.e. a negative colour gradient. The measured colour gradients of the complete sample of ELHCs are given in Col. 7 of Table 1. They have been determined from a linear least-squares fit to the observations. The table shows that among the fourteen newly presented ELHCs in this paper (ELHC 8 to 21) there are six ELHCs exhibiting a negative colour gradient. The remaining eight stars have a positive colour gradient, except ELHC 21 which shows a nearly grey variability.

We see therefore that the percentage of stars having a negative colour gradient is less than anticipated in LBD, where it was argued that a bluer-when-fainter behaviour is more likely to be observed at the distance of the LMC, provided that the inferred scattered blue light originates in a spherical nebula or in a not edge-on CS disk.
We would like to note that the measured colour gradients exclude that the variations are caused by a variable $\mathrm{H} \alpha$ emission line. $\mathrm{H} \alpha$ variability is an observed phenomenon among PMS stars (e.g. Catala et al. 1999). However the EROS1 filter system is such, that the $\mathrm{H} \alpha$ emission influences the flux measured in the $R_{\mathrm{E}}$ filter exclusively. Hence a change in its flux causes both the colour and $R_{\mathrm{E}}$ to change by the same amount. One thus expects a colour gradient $\mathrm{d}\left(B_{\mathrm{E}}-R_{\mathrm{E}}\right) / \mathrm{d} R_{\mathrm{E}}=1$. None of the ELHCs has the predicted colour gradient. Moreover, if we fold the EROS1 $R_{\mathrm{E}}$ filter with the spectrum of an early Btype star of which we increase the equivalent width of $\mathrm{H} \alpha$ from $80 \AA$ to $160 \AA$ we measure a change in the $R_{\mathrm{E}}$-magnitude of only $\Delta R_{\mathrm{E}} \sim 0.05^{\mathrm{m}}$. This is much smaller than the observed amplitude.

\subsubsection{The brightness state}

The time a star spends at a certain brightness level was determined by binning the magnitude measurements for every ELHC (see Parenago 1954). The size of the bins was chosen equal to the average uncertainty in the measurements. The qualification is meaningful only if the typical time scale of variability is much shorter than the 120 days of observations. For instance the classification of the light curve of ELHC 21 in terms of its brightness state is not very useful. The same applies to ELHC 15, 17, 20. One would expect that if variable dust obscuration is the dominant mechanism causing the variability of the ELHCs, the star will be preferably in a state of maximum brightness, provided that the average time for a single dust cloud to pass through the line of sight to the star is less than half the time span of the EROS1 light curve, i.e. less than 60 days. For Galactic UXors the characteristic time scale is on the order of days to weeks (Herbst \& Shevchenko 1999).

The determined brightness states for the ELHCs are given in Col. 8 of Table 1. The light curves of the stars have been given the classification according to the brightness state in which they have spent the longest period during the 120 days of the EROS1 observations. We notice that those ELHC, which have a positive colour gradient are never characterized by a "faint" state. This implies that these objects preferably have brightness decreases with respect to their average brightness. On the other hand ELHCs with a negative colour gradient are never characterized by a "bright" state. This indicates that these objects preferably have brightness increases with respect to their average brightness.

\subsection{The derivation of the ELHC stellar parameters}

The stellar parameters of ELHC 2 to 7 have been derived by LBD from optical spectra and the energy distributions. It was shown that a reasonable correspondence exists between the values of $L$ and $T_{\text {eff }}$ derived in this way and derived only from photometry, because the ELHCs were found to have only small CS extinction at brightness maximum: $-0.16 \lesssim E(B-V)_{\mathrm{CS}} \lesssim$ 0.11 . Small and even negative CS extinction values can be expected when a substantial fraction of the total flux is scattered radiation. 


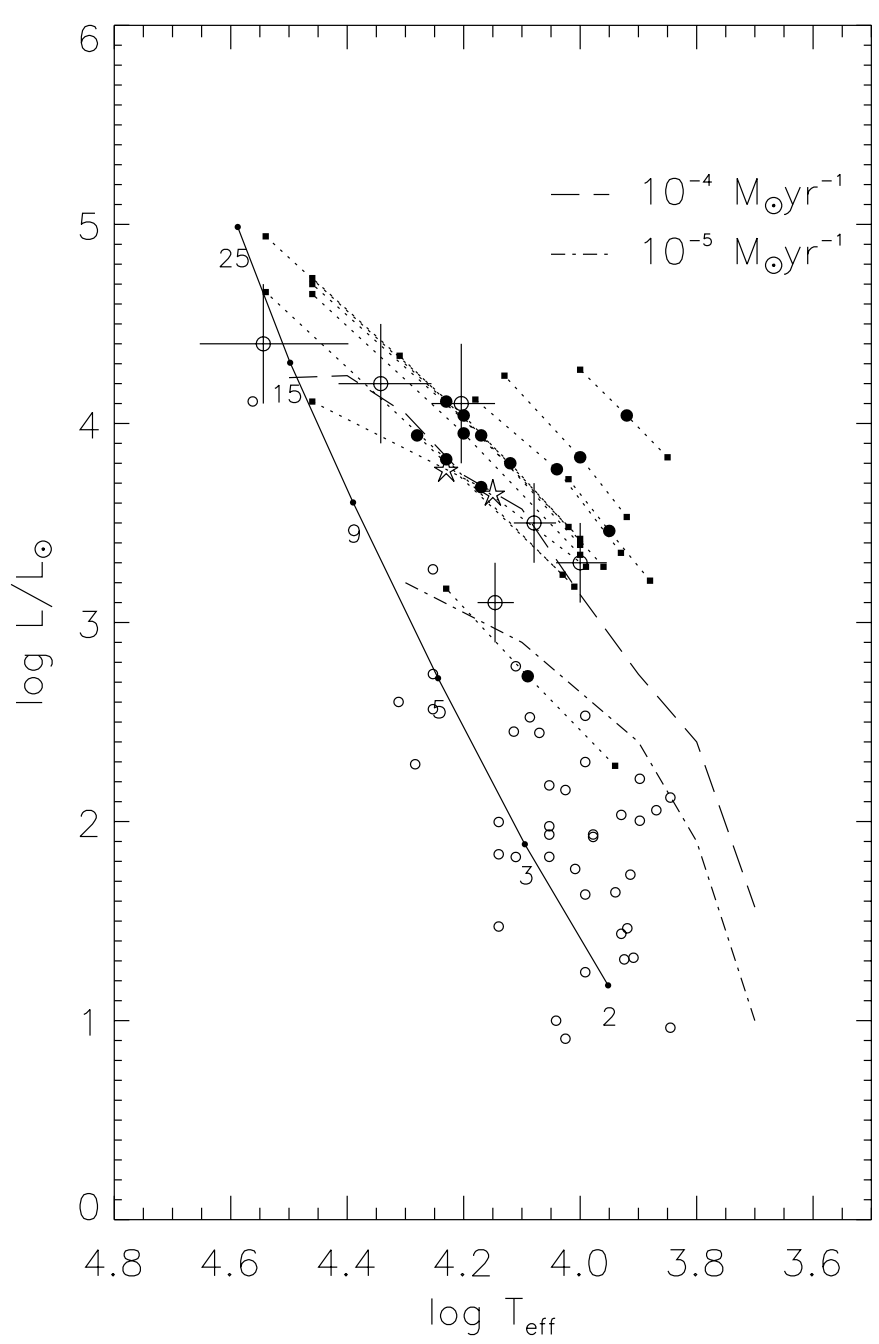

Fig. 5. A preliminary HR-diagram of the stars reported in this paper (filled circles and 2 open asterisks), based on their magnitudes and colours. The open circles are ELHC 2 to 7 from LBD. Indicated are Galactic HAeBe stars (small open circles) from Berrilli et al. (1992). The Main-Sequence with corresponding masses is indicated by a full line. The dashed-dotted and the dotted lines indicate two predicted birthlines from Palla \& Stahler $(1993,1994)$ for a mean mass accretion rate of $10^{-5} M_{\odot} \mathrm{yr}^{-1}$ and $10^{-4} M_{\odot} \mathrm{yr}^{-1}$ respectively.

Therefore we will estimate the range in stellar parameters for ELHC 8 to 21, for which only EROS1 photometry is available. We will use three possible values for the total extinction: (1) zero extinction, (2) only IS extinction with $E(B-$ $V)=0.17$, (3) IS extinction plus a maximum CS extinction of $E(B-V)_{\mathrm{CS}}=0.10$.

Following LBD, we use the magnitudes and colours of the object when it is in its brightest phase. We assume therefore that dust obscuration is the mechanism which causes the variability, as proposed for the ELHCs. We adopt the transformation equations between the EROS1 filter system and the Johnson system (see also LBD). We applied the calibration of $T_{\text {eff }}$ and the bolometric correction for luminosity class III stars (Schmidt-Kaler $1982)$ to the resulting three estimates of $(B-V)_{0}$ for every object. Extinction due to dust with $R_{\mathrm{V}}=3.0$ and a LMC distance modulus of 18.50 was adopted (for a recent discussion about the LMC distance determination, see Walker 1999).

The resulting stellar parameters for the adopted extinction values are listed in Table 2, the corresponding location in the HR-diagram is shown in Fig. 5.

\subsection{The location in the HR-diagram}

The Main Sequence in the HR-diagram is presented by the full line, with Zero Age Main Sequence masses indicated. The range of parameters derived for ELHC 8 to 21 and for ELHC 1 (no spectrum for this star was presented in LBD) from Table 2 are connected by the dotted lines, which are bounded by small filled squares. The filled squares correspond to zero total extinction and maximum total extinction. The filled circles indicate the stellar parameters when only an IS extinction correction towards the LMC bar is applied. The range in luminosity is predominantly due to the sharp increase in bolometric correction for the early type stars. Two objects in Fig. 5 are presented by large open asterisks, viz. ELHC 16 and ELHC 20. These two stars are extremely blue and no physical colours could be derived in applying the two adopted extinction values. It may indicate a lower foreground extinction towards these stars or a dominant scattering contribution. These objects are presented without any extinction correction (see Table 2). The stellar parameters of the six ELHCs derived from spectroscopy (LBD) are the open circles. The figure shows that the location in the HR-diagram of the newly discovered LMC HAeBes follows the distribution of the ELHCs discussed in LBD.

We compare in Fig. 5 the location of the ELHCs in the HR-diagram with the stellar parameters derived for Galactic HAeBe stars (small open dots) by Berrilli et al. (1992). Note that the luminosity of the Galactic HAeBe stars is smaller than the dashed-dotted line, that represents the birthline of Palla \& Stahler $(1993,1994)$. The birthline corresponds to the predicted stellar parameters of a young stellar object which has terminated the proto-stellar mass accretion phase and will therefore be optically visible for the first time. The young object enters its Pre-Main Sequence contraction phase towards the Main Sequence. The location of the birthline in the HR-diagram depends on the rate at which the proto-stellar mass accretion process proceeds. The Galactic HAeBe stars from Berrilli et al. (1992) are compatible with a birthline corresponding to a mass accretion rate of $10^{-5} M_{\odot} \mathrm{yr}^{-1}$. In contrast, the more luminous nature of the the sample of LMC HAeBe candidates would indicate a higher mass accretion rate of $\lesssim 10^{-4} M_{\odot} \mathrm{yr}^{-1}$ (dashed line). Note that the (dotted) paths traced out by three adopted values for the total extinction of the ELHCs is approximately parallel to the birthline of a mass accretion rate of $10^{-4} M_{\odot} \mathrm{yr}^{-1}$. To determine a different accretion rate at lower metallicities, spectra of the ELHC stars are needed to put them accurately in the HR-diagram.

We conclude that the derived estimates for the stellar parameters locates nearly all ELHCs in an HR-diagram above the birthline of a mass accretion rate of $10^{-5} M_{\odot} \mathrm{yr}^{-1}$ except for two objects, viz. ELHC 7 and ELHC 9. We will show below that this is possibly not due to an observational bias, if the amplitude and 
Table 1. The parameters of the varying light curves of the HAeBe candidates, see text for details on the different parameters.

\begin{tabular}{|c|c|c|c|c|c|c|c|c|}
\hline $\begin{array}{l}\text { Star } \\
(1)\end{array}$ & $\begin{array}{c}<B_{\mathrm{E}}> \\
(2)\end{array}$ & $\begin{array}{c}<B_{\mathrm{E}}-R_{\mathrm{E}}> \\
\text { (3) }\end{array}$ & $\begin{array}{l}\Delta B_{\mathrm{E}} \\
(4)\end{array}$ & $\begin{array}{l}t_{\text {linear }}^{(1)} \\
(5)\end{array}$ & $\begin{array}{l}t_{\text {constant }}^{(1)} \\
\text { (6) }\end{array}$ & $\begin{array}{c}\mathrm{d}\left(B_{\mathrm{E}}-R_{\mathrm{E}}\right) / \mathrm{d} R_{\mathrm{E}} \\
(7)\end{array}$ & $\begin{array}{l}\text { Light Curve } \\
\text { (8) }\end{array}$ & $\begin{array}{c}\text { FAP } \\
(9)\end{array}$ \\
\hline ELHC 1 & 15.35 & -0.05 & 0.19 & 10 & 5 & $-0.19 \pm 0.04$ & faint & 0.015 \\
\hline ELHC 2 & 15.07 & 0.02 & 0.33 & 5 & 5 & $-0.32 \pm 0.03$ & average & 0.014 \\
\hline ELHC 3 & 15.66 & -0.01 & 0.25 & 5 & 5 & $-0.14 \pm 0.03$ & average & 0.049 \\
\hline ELHC 4 & 14.79 & 0.08 & 0.26 & 30 & $<5$ & $-0.22 \pm 0.05$ & average & 0.014 \\
\hline ELHC 5 & 15.63 & -0.01 & 0.14 & 10 & 30 & $-0.26 \pm 0.04$ & faint & 0.048 \\
\hline ELHC 6 & 15.84 & 0.11 & 0.22 & 15 & 5 & $+0.07 \pm 0.03$ & bright & 0.030 \\
\hline ELHC 7 & 16.95 & 0.06 & 0.45 & 15 & 15 & $+0.07 \pm 0.03$ & bright & 0.018 \\
\hline ELHC 8 & 15.28 & 0.08 & 0.16 & 30 & 10 & $+0.66 \pm 0.15$ & average & 0.020 \\
\hline ELHC 9 & 17.99 & 0.16 & 0.50 & 20 & - & $+0.40 \pm 0.09$ & bright & 0.016 \\
\hline ELHC 10 & 14.06 & 0.42 & 0.36 & 10 & 10 & $+0.28 \pm 0.06$ & bright & 0.014 \\
\hline ELHC 11 & 15.26 & 0.15 & 0.25 & - & - & $-0.24 \pm 0.02$ & faint & 0.025 \\
\hline ELHC 12 & 15.87 & 0.05 & 0.22 & 5 & 15 & $+0.58 \pm 0.10$ & bright & 0.020 \\
\hline ELHC 13 & 15.36 & 0.13 & 0.22 & 15 & - & $+0.45 \pm 0.11$ & average & 0.020 \\
\hline ELHC 14 & 15.21 & 0.05 & 0.31 & 10 & 5 & $+0.11 \pm 0.04$ & average & 0.019 \\
\hline ELHC 15 & 15.58 & 0.32 & 0.17 & 40 & - & $-0.16 \pm 0.02$ & - & 0.032 \\
\hline ELHC 16 & 15.31 & -0.13 & 0.15 & 10 & 15 & $-0.24 \pm 0.04$ & faint & 0.033 \\
\hline ELHC 17 & 14.79 & 0.21 & 0.15 & - & - & $-0.13 \pm 0.02$ & - & 0.064 \\
\hline ELHC 18 & 15.47 & 0.03 & 0.19 & 10 & 5 & $-0.40 \pm 0.02$ & faint & 0.033 \\
\hline ELHC 19 & 15.89 & 0.05 & 0.19 & 10 & 5 & $+0.37 \pm 0.06$ & average & 0.025 \\
\hline ELHC 20 & 15.44 & -0.16 & 0.18 & - & - & $-0.27 \pm 0.04$ & - & 0.025 \\
\hline ELHC 21 & 15.23 & 0.04 & 0.21 & 25 & $>40$ & $-0.03 \pm 0.05$ & - & 0.010 \\
\hline
\end{tabular}

(1) In days.

time scale of the variations of less luminous HAeBe candidates would be similar to the luminous ELHCs (see Sect. 8).

\section{Interpretation of the ELHC light curves in terms of dust obscuration}

The optical variability of HAeBes can be understood in terms of obscuration of the star by dust clouds within the circumstellar environment. We will describe the features observed in the light curves of the ELHCs with a number of parameters and interpret these parameters in terms of the obscuration model. It will allow us to make quantitative estimates of the diameter of an individual obscuring cloud, its orbital distance and its mass.

The geometry of the model and the parameters are exemplified in Fig. 6. It consists of a star (with diameter $D_{\text {star }}$ ), a dust cloud (with a diameter $D_{\text {cloud }}$ and a transverse velocity $V_{\text {cloud }}$ ), and a blue scattering nebula. We will assume that the dust clouds are of homogeneous optical depth and orbit the star in a Keplerian orbit. The scattering nebula is assumed to be spherically symmetric and much larger than the obscuring cloud.

\subsection{Two time scales of the light curves}

We define two time scales for features observed in the light curve:

Linear time scale: the linear time scale parameter $\left(t_{\text {linear }}\right)$ is defined as the mean time scale of constant brightness increase or decrease of a light curve. It represents the amount of time it takes for a whole cloud to move out of or into the line of sight to the star. This time scale depends on the velocity of the obscuring cloud $V_{\text {cloud }}$. The time scale reaches a maximum when the diameter of the cloud $D_{\text {cloud }}$ is equal to or larger than the diameter of the star $D_{\text {star }}$. Then the linear time scale will be $t_{\text {linear }} \simeq D_{\text {star }} / V_{\text {cloud }}$.

Constant time scale: we define the constant time scale parameter $\left(t_{\text {constant }}\right)$ as the mean time scale with no variation in a light curve during a brightness minimum (within the uncertainty of the measurements). It is indicative of the amount of time it takes for the cloud to traverse the stellar disk. It depends on the transverse velocity of the cloud and its diameter as well as the diameter of the stellar disk. The constant parameter will be equal to $t_{\text {constant }} \simeq\left(\left|D_{\text {star }}-D_{\text {cloud }}\right|\right) / V_{\text {cloud }}$.

If we would consider the case of a cloud size smaller than that of the star, the two time scales will be equal when the diameter of the cloud is half the diameter of the star. In the opposite case of a cloud size larger then that of the star, the two time scales will be equal when the diameter of the cloud is twice the diameter of the star. The observed time scales of the ELHC light curves are tabulated in Cols. 5 and 6 of Table 1. Though the time the star spends in a dim state ( $t_{\text {constant }}$ ) is on the average less then the time it takes for a dust cloud to obscure the star, they are both in the order of days to tens of days. This leads us to conclude that the linear sizes of cloud and star should be of the same order.

\subsection{The amplitude of variation}

The full peak to peak amplitude of variability of the ELHCs in the $B_{\mathrm{E}}$ magnitude is given in Col. 4 of Table 1. The ELHCs have an amplitude in $B_{\mathrm{E}}$ of a few tenths of a magnitude. The faintest 
Table 2. The stellar parameters for the ELHCs derived for different extinction corrections, with an absolute to relative extinction factor $R_{\mathrm{V}}=3$. The parameters were derived when the star was at its brightest phase.

\begin{tabular}{|c|c|c|c|c|c|c|c|c|c|}
\hline Star & $V_{\mathrm{J}}$ & $M_{\mathrm{V}}$ & $B-V$ & $\begin{array}{r}\log \left(T_{\text {eff }}\right) \\
E(B)\end{array}$ & $\begin{array}{l}\log \left(L_{\odot}\right) \\
=0\end{array}$ & $\begin{array}{r}\log \left(T_{\text {eff }}\right. \\
E(B-\end{array}$ & $\begin{array}{l}\log \left(L_{\odot}\right) \\
=0.17\end{array}$ & $\begin{array}{c}\log \left(T_{\text {eff }}\right) \\
E(B-1\end{array}$ & $\begin{array}{l}\log \left(L_{\odot}\right) \\
=0.27\end{array}$ \\
\hline ELHC 1 & 15.41 & -3.11 & -0.02 & 4.00 & 3.28 & 4.17 & 3.68 & 4.46 & 4.11 \\
\hline ELHC 8 & 15.19 & -3.31 & -0.02 & 4.00 & 3.39 & 4.17 & 3.94 & 4.46 & 4.70 \\
\hline ELHC 9 & 17.75 & -0.75 & 0.06 & 3.94 & 2.28 & 4.09 & 2.73 & 4.23 & 3.17 \\
\hline ELHC 10 & 13.80 & -4.70 & 0.30 & 3.85 & 3.83 & 3.92 & 4.04 & 4.00 & 4.27 \\
\hline ELHC 11 & 15.05 & -3.45 & 0.10 & 3.93 & 3.35 & 4.04 & 3.77 & 4.18 & 4.12 \\
\hline ELHC 12 & 15.79 & -2.71 & -0.06 & 4.03 & 3.24 & 4.28 & 3.94 & 4.28 & 3.94 \\
\hline ELHC 13 & 15.24 & -3.26 & 0.04 & 3.96 & 3.28 & 4.12 & 3.80 & 4.31 & 4.34 \\
\hline ELHC 14 & 15.09 & -3.41 & -0.05 & 4.02 & 3.48 & 4.23 & 4.11 & 4.54 & 4.94 \\
\hline ELHC 15 & 15.32 & -3.18 & 0.23 & 3.88 & 3.21 & 3.95 & 3.46 & 4.02 & 3.72 \\
\hline ELHC 16 & 15.27 & -3.23 & -0.15 & 4.15 & 3.64 & 0.00 & 0.00 & 0.00 & 0.00 \\
\hline ELHC 17 & 14.58 & -3.92 & 0.12 & 3.92 & 3.53 & 4.00 & 3.83 & 4.13 & 4.24 \\
\hline ELHC 18 & 15.32 & -3.18 & -0.03 & 4.00 & 3.34 & 4.20 & 3.95 & 4.46 & 4.65 \\
\hline ELHC 19 & 15.79 & -2.71 & -0.04 & 4.01 & 3.18 & 4.23 & 3.82 & 4.54 & 4.66 \\
\hline ELHC 20 & 15.45 & -3.05 & -0.22 & 4.23 & 3.76 & 0.00 & 0.00 & 0.00 & 0.00 \\
\hline ELHC 21 & 15.11 & -3.39 & -0.02 & 4.00 & 3.42 & 4.20 & 4.04 & 4.46 & 4.73 \\
\hline
\end{tabular}

stars show the largest amplitudes, while the bright stars did not display variations larger than $\Delta B_{\mathrm{E}} \simeq 0.35^{\mathrm{m}}$ during the observation of 120 days. This could imply that there is a correlation between brightness and amplitude of variation, and possibly a relation between the amount of scattered light from the nebula and the spectral type of the star.

One can express the amplitude of variation in terms of the (unresolved) nebula (indicated by the superscript "N", see equations below) and the much smaller obscuring cloud ("CL") at the wavelength under consideration. The optical depth of the nebula, between the star and its outer edge is $\tau_{\text {scat }}^{\mathrm{N}}$ for scattering, and $\tau_{\mathrm{abs}}^{\mathrm{N}}$ for absorption. The total optical depth (scattering and absorption) of the cloud is $\tau_{\mathrm{ext}}^{\mathrm{CL}}$. The intrinsic stellar radiation is $F_{0}^{*}$. If there is no obscuring cloud, the observed flux from the nebula plus star will be

$F^{\mathrm{obs}}=F_{0}^{*} \mathrm{e}^{-\tau_{\mathrm{abs}}^{\mathrm{N}}}$,

which consists of the intrinsic flux of the star attenuated by the column depth of the nebula in the line of sight, $F^{*}=$

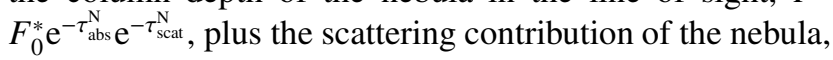

$F^{\mathrm{N}}=F_{0}^{*} \mathrm{e}^{-\tau_{\text {abs }}^{\mathrm{N}}}\left(1-\mathrm{e}^{-\tau_{\text {scat }}^{\mathrm{N}}}\right)$.

When a cloud transits the star the stellar contribution to the observed flux will diminish due to the extinction of the cloud, i.e.

$F^{*}=F_{0}^{*} \mathrm{e}^{-\tau_{\text {abs }}^{\mathrm{N}}} \mathrm{e}^{-\tau_{\text {scat }}^{\mathrm{N}}} \mathrm{e}^{-\tau_{\text {ext }}^{\mathrm{CL}}}$

while the nebular contribution remains the same, as it is much larger than the cloud. The total observed flux will then read

$F^{\mathrm{obs}}=F_{0}^{*}\left(\mathrm{e}^{-\tau_{\mathrm{abs}}^{\mathrm{N}}}-\mathrm{e}^{-\tau_{\mathrm{abs}}^{\mathrm{N}}} \mathrm{e}^{-\tau_{\mathrm{scat}}^{\mathrm{N}}}+\mathrm{e}^{-\tau_{\mathrm{abs}}^{\mathrm{N}}} \mathrm{e}^{-\tau_{\mathrm{scat}}^{\mathrm{N}}} \mathrm{e}^{-\tau_{\mathrm{ext}}^{\mathrm{C}}}\right)$

in which the scattering contribution of the obscuring cloud to the total scattered radiation is neglected. Now it is easy to show that the observed brightness variations will be given by

$\Delta B_{\mathrm{E}}=-2.5 \log \left(1-\mathrm{e}^{-\tau_{\mathrm{scat}}^{\mathrm{N}}}\left(1-\mathrm{e}^{-\tau_{\mathrm{ext}}^{\mathrm{CL}}}\right)\right)$.
The equation states that in the case of a small contribution of the scattering nebula $\left(\tau_{\text {scat }}^{\mathrm{N}} \ll 1\right)$ the amplitude will equal the optical depth of the obscuring cloud. This will then be the minimum optical depth for the obscuring cloud. On the other hand, if the obscuring cloud has a large optical depth $\left(\tau_{\text {ext }}^{\mathrm{CL}} \gg 1\right)$ the amplitude of the photometric variations gives a maximum scattering optical depth of the nebula. From the data in Col. 4 of Table 1 we conclude that the minimum optical depth of the cloud is in the order of a few tenths and the maximum scattering optical depth of the nebula is about 1 to 2 .

\subsection{Quantitative properties of the CS environment}

With the help of the conclusions drawn in the previous two subsections, we derive "first order" estimates of the physical conditions governing the observed variations for a typical ELHC with a mass and radius $M=10 M_{\odot}, R=10 R_{\odot}$.

First we estimate the dust mass contained in a spherical obscuring cloud. The minimum dust mass is determined by its minimum optical depth. For this mass estimate we use a standard MRN dust mixture (Mathis et al. 1977), together with the tabulated dust absorption efficiency for $0.01 \mu \mathrm{m}$ astronomical silicate particles by Draine (1985) and a value of 0.3 for the amplitude of variation. This leads to a minimum dust mass $\sim 10^{20} \mathrm{~g}$ for one obscuring cloud.

The maximum optical depth of the cloud is poorly constrained. To deduce its value from the amplitude of variation, using Eq. (5), the exact relative contribution of the scattering nebula has to be known. In principle this can be derived using the colour information of the ELHC light curves, combined with the scattering and absorbing properties of the dust in the CS environment, characterized by some geometry. This will be the subject of a forthcoming paper. However we do not expect the obscuring clouds to be extremely dense for the following reason. The clouds are probably more or less spherically distributed in the CS environment of $\mathrm{HAeBe}$ stars, 


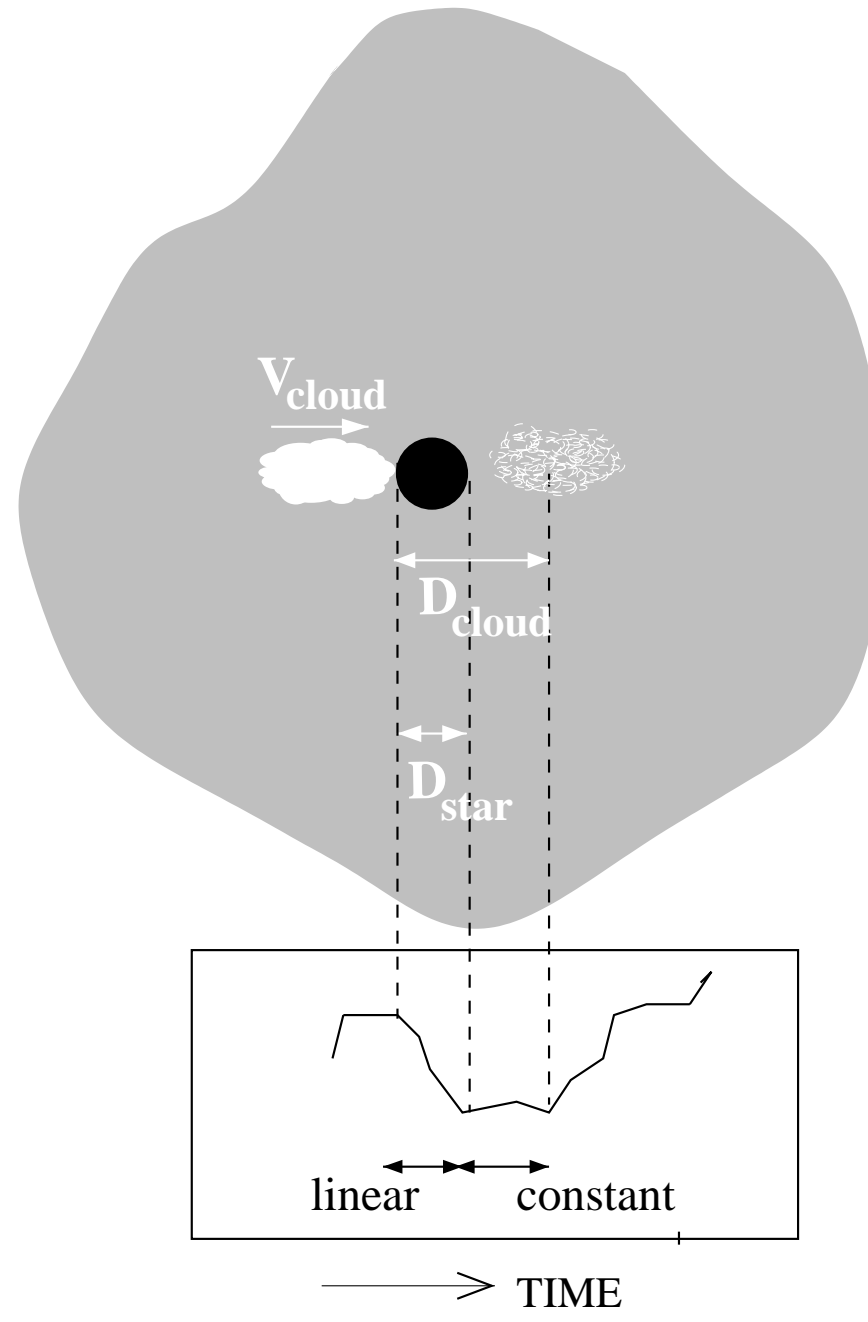

Fig. 6. Interpreting the optical light curves of the ELHCs with a simple model, representing the star, an obscuring dust cloud and the scattering nebula. The configuration is not to scale.

because the photometric variation seems to be a generic feature among HAeBes (Herbst \& Shevchenko 1999). Furthermore, the HAeBe stars seem to vary continuously, which argues for a system consisting of a star surrounded by a spherical dust shell containing many clouds. If the clouds would be very dense, the stellar radiation would be severely attenuated and reddened by the opaque dust shell. The scattered radiation of the nebula would also be redder, for the nebula is at much larger radii from the central star than the obscuring clouds and would thus scatter reddened stellar radiation. This implies a large extinction correction. However in most cases the extinction towards $\mathrm{HAeBe}$ stars is low (Van den Ancker et al. 1998), making dense obscuring dust clouds very unlikely. Therefore we will estimate the maximum optical depth of the cloud to be the maximum possible total optical depth of the scattering nebula, accounting for the mean amplitude of the variations of 0.3 , i.e. $\tau_{\text {scat }}^{\mathrm{N}}$ is 1.4. Then, from the results of Wolfire \& Cassinelli (1986) (their Fig. 1) for a MRN mixture, we can estimate the total absorption optical depth of the nebula, if the grain properties of the obscuring cloud and scattering nebula are the same. They show that the scattering opacity is about 5 times the absorption opacity, hence the absorption optical depth is $\sim 0.3$. Note that this value is consistent with the low $E(B-V)_{\mathrm{CS}}$ values $(\leq 0.11)$ found for the ELHCs in LBD, adopting the standard value for the absolute to selective extinction of 3.1. Thus we expect the maximum optical depth of the cloud to be $\tau_{\text {ext }}^{\mathrm{CL}} \lesssim 1.7$. Then the maximum dust mass contained in the obscuring clouds will be $\sim 10^{21} \mathrm{~g}$.

For Galactic HAeBes column densities of obscuring clouds have been calculated for Galactic UXOrs by Voshchinnikov \& Grinin (1991). They derived a dust mass of the central core of the obscuring cloud of $1.6 \times 10^{20} \mathrm{~g}$ for WW Vul, during a deep photometric minimum. Our estimates are of the same order of magnitude.

An interesting question related to the overall density stratification of the CS material is whether the obscuring clouds are self gravitating or whether the brightness variations are caused by more or less stochastic density fluctuations in the CS environment. An orbiting dust cloud will be tidally disrupted when its mass is less than $2 M_{*}\left(R_{\text {cloud }} / a_{\text {kep }}\right)^{3}$, where $a_{\text {kep }}$ is the Keplerian orbital distance and $R_{\text {cloud }}$ the radius of the cloud. Using the expression between $V_{\text {cloud }}$ and $t_{\text {linear }}$ from Sect. 6.1, the velocity of a dust cloud and its Keplerian orbit is set. For the ELHCs the orbital distances of the clouds range between 8.5 AU and 400 AU. Note that for the expected effective temperatures of the ELHCs, these distances are beyond the dust sublimation point for dust grains in thermal equilibrium. The orbital distances translate to minimum tidally stable masses of $2 \times 10^{22} \mathrm{~g}$ and $6 \times 10^{27} \mathrm{~g}$ for the longest and the shortest values of $t_{\text {linear }}$ respectively. This means that stars displaying a time scale of variation longer than $\sim 30$ days can have tidally stable clouds. These stars can have dust cloud masses a few times $10^{20} \mathrm{~g}$ for a gas to dust mass ratio of 100 . However only three ELHCs appear to have such long time scales of variation.

We would like to point out that these rough estimates depend very sensitively on the dust properties. Larger average silicate particles than the MRN mixture have been found for Galactic HAeBes through more detailed calculations (Voshchinnikov et al. 1996; Voshchinnikov et al. 1995; Rostopchina et al. 1997). If this is the case, the optical depth cloud mass can decrease by orders of magnitude and none of the obscuring clouds will be stable. Therefore we tentatively conclude that the photometric variations are more likely to be stochastic density fluctuations of the inhomogeneous CS environment.

\subsection{The influence of the nebula}

The colour behaviour of the stars in this model will also depend on the properties of the inferred nebula. The way the colour varies is set by the ratio of the flux of the blue scattered nebular light to the stellar flux which reddens due to the obscuring dust cloud. In Col. 7 of Table 1 we presented the measured colour gradient $\mathrm{d}\left(B_{\mathrm{E}}-R_{\mathrm{E}}\right) / \mathrm{d} R_{\mathrm{E}}$ of the ELHCs. When the colour gradient is negative the star displays the bluer when fainter behaviour. More than half of the ELHCs have a negative colour gradient. This fact argues for a significant contribution of the scattering nebula to the EROS1 photometry of these ELHCs. 
Indeed, using Eq. (2), for the maximum contribution of the nebula $\left(\tau_{\text {scat }}^{\mathrm{N}}=1.4\right)$ and an amplitude of variation of 0.3 , one finds that the nebula contributes 4 times more light to the observed flux than the star.

\section{Quantitative comparison between MPG and AoV methods for the detection of variable stars}

In Sect. 3 we stated that the AoV phase binning method is expected to be more efficient in detecting irregular variable stars than the MPG Fourier harmonics method. This explains partly the fact that with AoV more $\mathrm{HAeBe}$ candidates were found then in LBD. In this section we elaborate and quantify the difference between the two methods.

\subsection{Comparison of false alarm probabilities}

The basis for identifying a variable (regular or irregular) star is the power spectrum produced by the period search method. A star is an accepted variable if the peak value of the power spectrum is above a certain threshold value at a certain trial period (which we empirically estimated for the EROS1 dataset to have value of 30, Sect. 3.1). Because the noise has a stochastic component, the threshold power due to noise has a specific probability.

In principle all period search methods deliver analytical probability distributions for the generated power at a trial period, assuming the data is pure noise. However in practice, such a distribution is best obtained using the empirical data (Schwarzenberg-Czerny 1998). Usually, using a Monte Carlo type simulation, synthetic light curves with an added noise component are generated to calculate the probability to find a certain value of the maximum power or higher. This distribution is the empirical false alarm probability (FAP) distribution (see Scargle 1982; Horne \& Baliunas 1986). Basically, a high value of the FAP means a high probability that the found power is due to noise.

To compare AoV with MPG we calculated the FAP distribution for both methods, in this case however using 2000 existing light curves of EROS1 stars. In this way we include all the possible contributing systematic errors inherent to the EROS1 data. Hence any light curve with low FAP produced in this way has a high probability to be intrinsically variable, well beyond the systematic noise of the EROS1 measurements and the white noise. Note that in calculating the FAP distributions for AoV and MPG, we do not exclude the light curves of the known variable stars. Hence, strictly speaking, it is not a FAP distribution, but it is the distribution one obtains exploring a large dataset of light curves of stars with unknown nature.

The difference in efficiency between the two methods is determined by comparing the FAPs of the maximum power produced by the light curves of the different kinds of known variable stars with both methods. It is shown in Fig. 7. Every panel constitutes the cumulative distribution of the FAP values for a different kind of variable, displaying both AoV FAPs (solid line) and MPG FAPs (dashed line).

In the upper left panel, one notices that AoV produces slightly lower FAPs than MPG and thus is more efficient
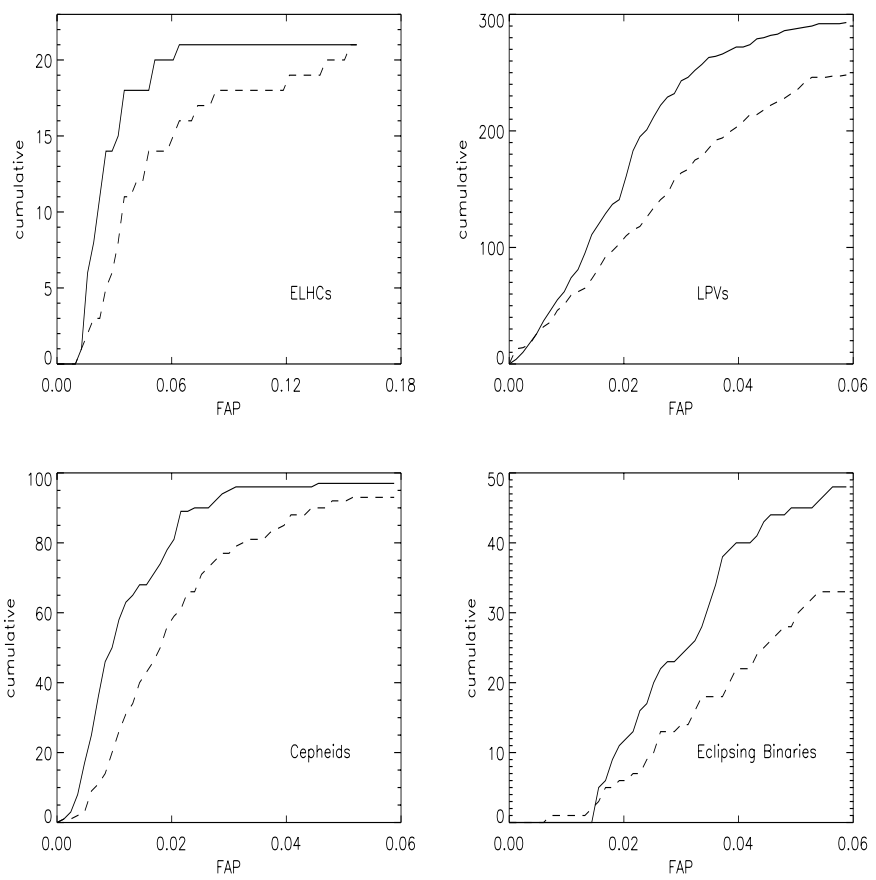

Fig. 7. Comparison of the cumulative distributions of the False Alarm Probabilities (FAP) generated with AoV method (full line) and MPG method (dashed line). Both regular as well as quasi-regular or irregular variables are detected with a higher efficiency when using AoV.

in identifying irregular and quasi-regular variables (ELHCs). However also for the regular variables as Cepheids and Eclipsing Binaries AoV produces lower FAPs. Hence, Fig. 7 illustrates that AoV is also slightly more efficient in finding regular variables from a large database of light curves than MPG. The FAP values generated by the ELHCs are larger than the FAP values found for the other three types of variables in Fig. 7. However the 21 ELHCs are found with a FAP lower than 0.06 . This means that the ELHCs are among the most variable (i.e. in terms of highest power) $6 \%$ of the total number of stars.

\subsection{Comparison of power spectra}

We compared the power spectra produced by AoV and MPG for two ELHCs. If AoV is more efficient in identifying irregular type variables and stars showing trends than MPG, then this difference could appear in the power spectra. Figure 8 displays the spectra of ELHC 14 and ELHC 15 for both methods. ELHC 14 has a quasi-regular type light curve, while ELHC 15 shows a downward trend in its light curve, with some variation between days 740 and 760 since JD $=2447862.5$ (see Fig. 2). We computed the power spectra using the same search parameters.

Figure 8 shows that both AoV and MPG power spectra of ELHC 14 show more features than the ones of ELHC 15. The peak value of the ELHC 14 AoV power spectrum occurs at a trial frequency of $0.049 \pm 0.004 \mathrm{day}^{-1}$ or a period 20.4 days. (The error estimate corresponds to the sigma of the Gaussian fitted to the peak, the adopted frequency step is 0.005.) This corresponds to the observed main time scale of variation seen in its light curve. The second highest peak is 

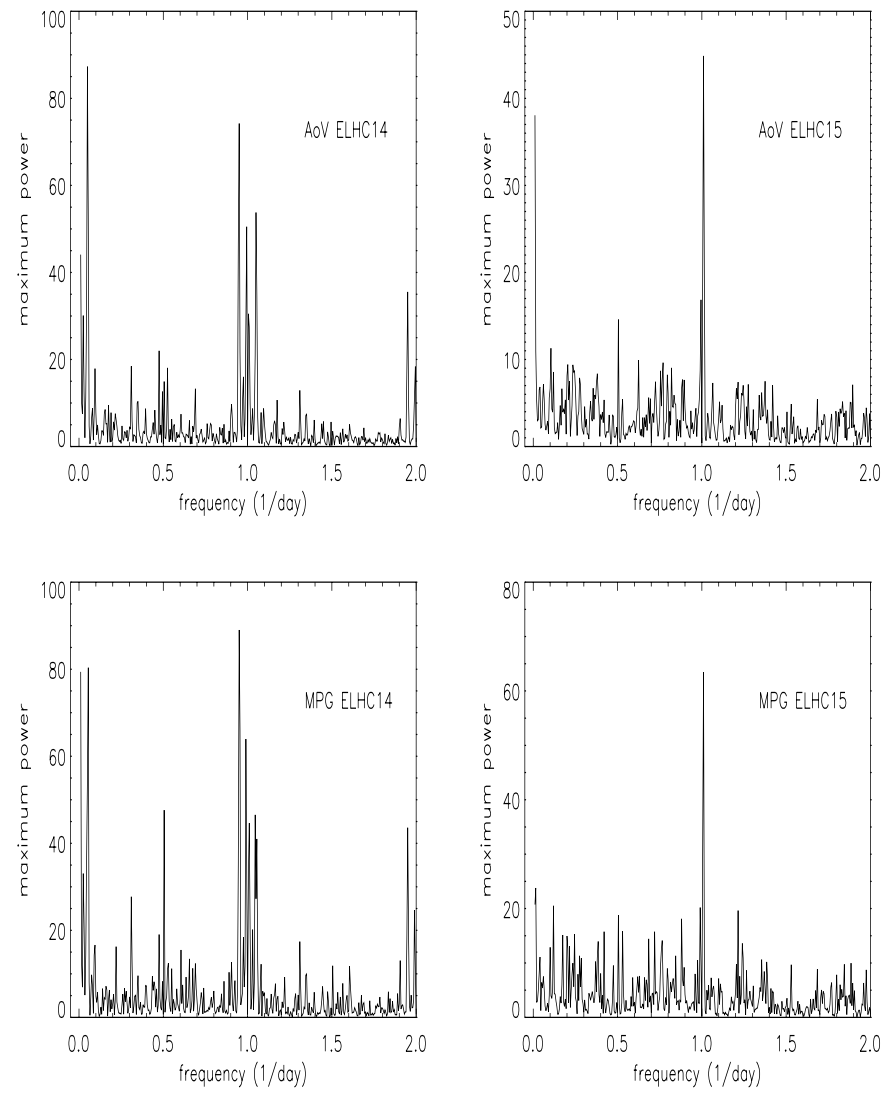

Fig. 8. Comparison of power spectra generated with AoV and MPG for ELHC 14 and ELHC 15.

at the frequency $0.94 \pm 0.004 \mathrm{day}^{-1}$, which corresponds to the 1 day minus 20.4 days in frequency space. For the ELHC 14 MPG power spectrum the peak value is at this same frequency of $0.94 \pm 0.004 \mathrm{day}^{-1}$. Strong aliasing is seen near a trial period of 1 day. The values of the maximum power produced at these periods by MPG and AoV are nearly equal. For ELHC 15 both methods produce strong power at aliasing period of 1 day. However AoV produces also a significant power at a trial frequency of $0.01 \pm 0.004 \mathrm{day}^{-1}$ or a period 100 days. It is on this time scale that the light curve of ELHC 15 shows the downward trend.

The reason these two stars were missed in LBD is that the main power produced by MPG for ELHC 15 is at the aliasing period of 1 day, whereas for ELHC 14 around $0.94 \pm$ $0.004 \mathrm{day}^{-1}$. The search in LBD was limited to objects with a most probable period of $>60$ days.

\section{The number of ELHCs}

We make a simple estimate of the total number of HAeBe candidate stars in the bar of the LMC, by testing how efficient the Analysis of Variance method is in detecting irregular variable stars. It addresses the question whether the detection of predominantly luminous ELHCs is related to a different variability (smaller amplitude, different time scale) of possibly less luminous HAeBe stars.

We chose 2000 existing EROS1 light curves of nonvariable stars (stars with low FAP) These stars cover a

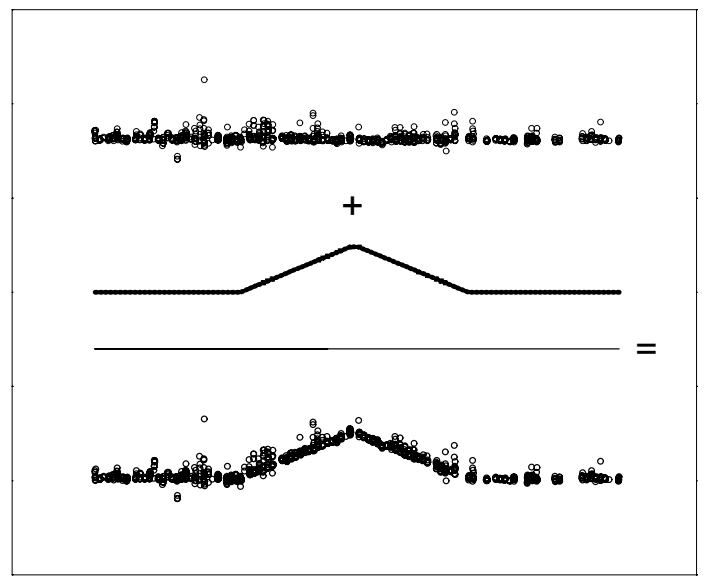

Fig. 9. The generation of synthetic light curves from an (observed) EROS1 noise signal of a non-variable star (top part) by adding a variable signal (middle part). The lower part is the resulting light curve for which the efficiency of the AoV method can be tested.

magnitude range from $14^{\mathrm{m}}$ to $19^{\mathrm{m}}$. They are representatives of the EROS1 dataset. We add to their light curves different types of signals. The signals were chosen such that they simulate some of the observed features of the ELHCs' brightness variations: different shapes (see Fig. 10), 5 different amplitudes per shape for 3 different time scales (10, 25 and 50 days). We applied the AoV algorithm and determined the number of detected "variables" according to the same threshold criterion as applied to the detection of ELHCs (Sect. 3.1). The efficiency of the AoV algorithm like in Fig. 10 is then simply defined as the fraction of detected "variables" to the total number of input stars (i.e. 2000).

We note that this is only a limited test of the efficiency of the AoV algorithm. A more thorough investigation of other signals is beyond the scope of this paper.

\subsection{Different signals}

An example of the AoV efficiency in detecting certain signals is plotted in Fig. 10. The type of signal has been indicated in the upper right corner of every panel. The first panel shows the efficiency of the AoV method for a periodic sinusoidal signal with a period of 10 days for 5 different amplitudes. The signal was applied covering the whole observing window of 120 days. We found only very minor differences in efficiency for sinusoids with different periods. The panel shows that for stars brighter than $16.5^{\mathrm{m}}$ the AoV-method will identify all regular variables with full peak to peak amplitudes larger than $0.1^{\mathrm{m}}$, i.e. most likely all Cepheids within the EROS1 database up to this amplitude limit have been detected. While up to a magnitude of $\sim 19^{\mathrm{m}}$ (i.e. the minimum average brightness of an RR Lyrae star), the AoV method will in principle detect $85 \%$ of variable stars with full amplitude of $0.2^{\mathrm{m}}$, though in practice crowding effects severely limits the detection efficiency.

The middle panel of Fig. 10 shows the efficiency of AoV in detecting non-periodic signals of a star whose light curve drops in brightness on a short time scale, while it otherwise remains constant (similar to ELHC 21). The right panel shows the 

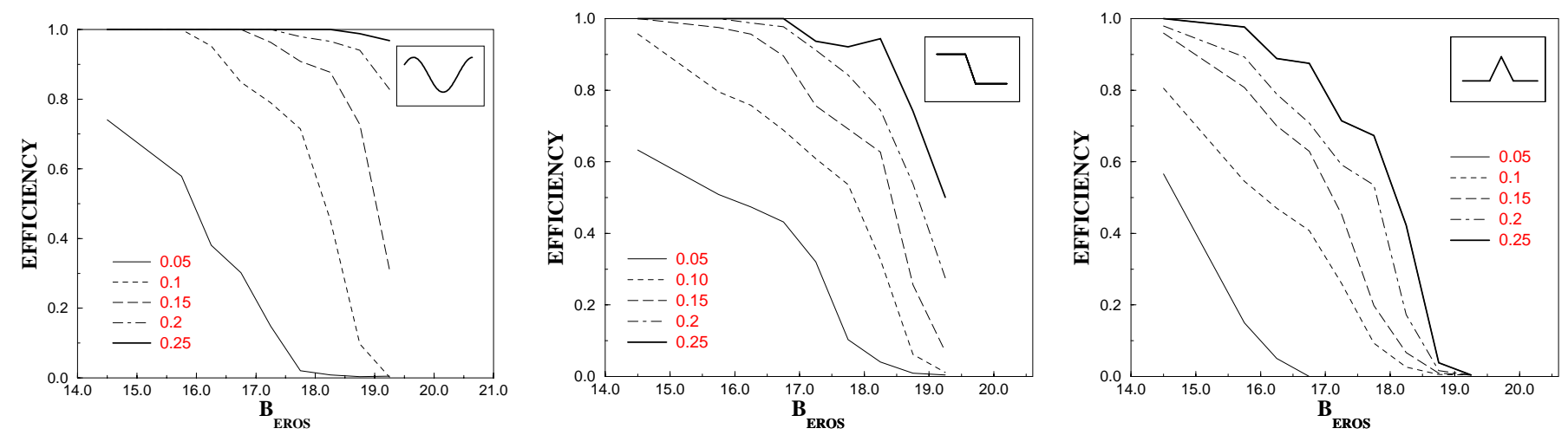

Fig. 10. The distributions of the detection efficiency for 5 different values of the amplitude of a signal (as indicated in the top right of each panel) as a function of the EROS1 $B_{\mathrm{E}}$ magnitude. In the left panel the efficiency is plotted for a sinusoidal signal, middle panel is an obscuration type signal and the right panel is an triangle is added with a time scale of 25 days.

sensitivity of the AoV-method to detect light curves, which have a symmetric triangle with a time scale of one fifth of the total observing window (i.e. 25 days) on top (like ELHC 1, ELHC 8, ELHC 16).

\subsection{Application to ELHCs}

In Fig. 11 we show the general results of the AoV efficiency in detecting a triangular shaped signal. We determined the $66 \%$ efficiency of a certain amplitude at three different time scales for the signal. The three plotted relations are for stars with different magnitudes and correspond to bins at median magnitudes of $14.5^{\mathrm{m}}, 16.25^{\mathrm{m}}, 17.75^{\mathrm{m}}$ in $B_{\mathrm{E}}$. The figure shows the obvious results that (1) it becomes more difficult to detect a signal at lower signal to noise ratios and (2) it becomes more difficult to detect a signal if the signal is present for a smaller time (a shorter time scale).

In Fig. 11 we also plotted the amplitude and time scale of the ELHCs with their average magnitude indicated. The plotted ELHC time scale is the most probable period as found by AoV. Note that ELHC 6 is the faintest of the plotted stars with a $B_{\mathrm{E}}$ of $15.84^{\mathrm{m}}$. All ELHCs are distributed well above the curve which would correspond to their limiting magnitude for a $66 \%$ detection probability. This implies that smaller amplitudes and/or smaller time scales could have been detected if these stars had existed in the EROS1 database. We conclude that our sample of ELHC stars selected on the variability criterion is not hampered by incompleteness up to a magnitude limit of $\sim 16.5^{\mathrm{m}}$ up to a minimum full amplitude of $0.2^{\mathrm{m}}$ and a minimum time scale of 20 days.

Consequently the sample of $\mathrm{HAeBe}$ candidates will be selected biased towards larger amplitudes and longer time scales of variations. Half of the ELHCs have their maximum peak in the AoV periodogram at 94 days or more, where we searched from 0.5 days to 100 days. Although these stars are also variable on shorter time scales, as evidenced by the light curves, the main power is produced by the overall variation of the ELHC light curve. The other half of the ELHCs are the stars with a strong signal on shorter time scale

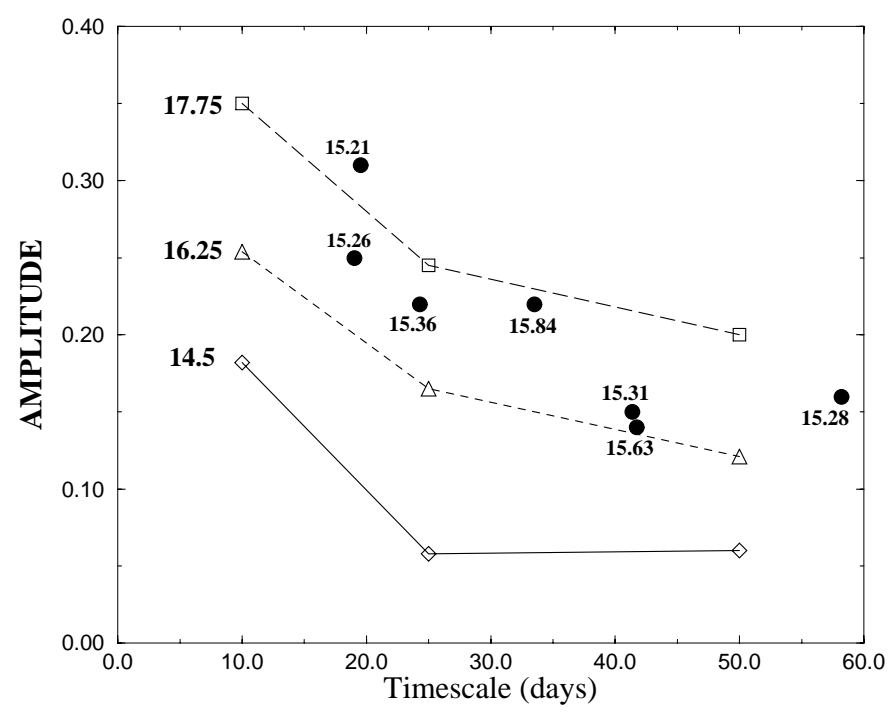

Fig. 11. The amplitude dependence of the detection at an efficiency of 0.66 as a function of time scale of an added triangle for different magnitudes (mentioned on the left of each curve in this panel), also plotted are ELHCs indicated with black dots and their average magnitude. It shows that fainter variable stars could easily have been detected if they existed in the database. For details see text.

(cf. ELHC 13, ELHC 16, ELHC 19). The shortest quasi-period found is 19 days (ELHC 11).

However if we want to identify typical Galactic HAeBe stars at the distance of the LMC on the basis of the optical variability criterion, the probability to detect such a star of amplitude of $0.25^{\mathrm{m}}$ is small (let alone the crowding difficulties) when this star is fainter than $18.5^{\mathrm{m}}$, i.e. an HAeBe star of $\sim 2.5 M_{\odot}$.

\section{Discussion and conclusions}

We have identified 14 new blue $\left(\left(B_{\mathrm{E}}-R_{\mathrm{E}}\right)<0.5\right)$ LMC stars with irregular photometric behaviour on time scales of days to tens of days, with amplitudes up to $0.5^{\mathrm{m}}$. The corresponding change in colour of the objects is characterized by either 
bluer-when-fainter or redder-when-fainter colour behaviour. Bluer-when-fainter objects were more often found in a brightness minimum than the redder-when-fainter ELHCs. In these respects they are very similar to the seven LMC HAeBe candidate stars studied in LBD, i.e. the ELHCs. Correlation of the location of all ELHCs with far infrared emission showed that the objects are preferably located on the rim of or in an IRAS $60 \mu \mathrm{m}$ excess emission region of the LMC bar. Where $60 \mu \mathrm{m}$ excess emission is indicative of star formation. This supports the notion that the objects are young.

Based on photometry, we have shown that all new ELHCs (except ELHC 9) occupy a region in the HR-diagram above the birthline corresponding to a mass accretion rate of $10^{-5} M_{\odot} \mathrm{yr}^{-1}$. Therefore, if confirmed these objects would constitute very luminous HAeBe stars, which might point to a fast proto stellar mass accretion rate.

A different dust to gas ratio in the LMC compared to the Galaxy is one of the suggestions explaining that the ELHCs are more luminous than Galactic HAeBes and above the predicted Galactic birthline for a mean accretion rate of $10^{-5} M_{\odot} \mathrm{yr}^{-1}$. Young LMC stars will be less embedded in dust than their Galactic counterparts. Subsequently accreting proto stars will be visible in an earlier stage. On the other hand the luminosity of the birthline depends on the mass accretion rate. If during the proto-stellar phase the mass accretion rate would be higher among LMC stars than Galactic stars, the birthline in the HRdiagram will shift to higher luminosities, enabling the detection of more luminous and more massive PMS stars.

Palla (private communication, see also Testi et al. 1998) has recently argued that the ELHCs may not be unique in this respect, because also some Galactic HAeBes can be above the canonical birthline of $10^{-5} M_{\odot} \mathrm{yr}^{-1}$. However the location in an HR-diagram of ELHCs of later type (ELHC 5 and 6, LBD) is not observed among Galactic HAeBe stars.

The generally accepted mechanism causing HAeBe stars to vary, is a changing amount of extinction of the stellar light due to a dusty inhomogeneous circumstellar environment. An interpretation of the light curves in terms of this model has been presented. To account for the bluer colours during brightness minima of ELHCs, a scattered light originating in a nebula was invoked. Based on light curve characteristics presented in Sect. 6, a simple calculation allowed us to give an estimate of the diameters of the obscuring clouds. The diameters are on the order of the stellar radius and the clouds orbit the star at a distance on the order of 10 to a few 100 AUs. We present arguments that the clouds are in fact stochastic density fluctuations, instead of self-gravitating dust clouds. We note however that the results depend strongly on the assumed dust properties.

We have shown the detection limits of the AoV method for different kinds of light curves and amplitudes in Sect. 8. For full amplitudes of variation larger than $0.2^{\mathrm{m}}$ and time scales larger than 20 days, the ELHC sample is fairly complete up to $B_{\mathrm{E}}=16.5^{\mathrm{m}}$. However the ELHCs are predominantly luminous stars. We found that less luminous objects compared to the majority of the ELHCs would have been detected with the AoV method, if these objects exhibit similar irregular variability as the luminous ELHCs. Therefore either these stars do not exist or have a different (smaller amplitude, different time scale)
Table A.1. The ELHCs in the bar of the LMC.

\begin{tabular}{lcll}
\hline \hline Star & $\begin{array}{c}\text { EROS1 name } \\
\text { (CCD-star) }\end{array}$ & $\begin{array}{l}\text { RA(2000) } \\
(\mathrm{h}, \mathrm{m}, \mathrm{s})\end{array}$ & $\begin{array}{l}\text { Dec(2000) } \\
\left({ }^{0},{ }^{\prime},{ }^{\prime \prime}\right)\end{array}$ \\
\hline ELHC 1 & $01-1603$ & 051815.30 & -693015.0 \\
ELHC 2 & $02-111$ & 051833.60 & -693942.0 \\
ELHC 3 & $08-830$ & 051629.50 & -691717.0 \\
ELHC 4 & $08-1335$ & 051718.30 & -692138.0 \\
ELHC 5 & $01-1414$ & 051821.30 & -693239.0 \\
ELHC 6 & $01-1457$ & 051818.70 & -693530.0 \\
ELHC 7 & $08-1005$ & 051639.50 & -692049.0 \\
ELHC 8 & $01-837$ & 051717.70 & -693334.2 \\
ELHC 9 & $01-749$ & 051706.20 & -693323.3 \\
ELHC 10 & $02-765$ & 051948.30 & -693909.6 \\
ELHC 11 & $02-915$ & 051836.20 & -693560.0 \\
ELHC 12 & $02-999$ & 051850.00 & -693521.2 \\
ELHC 13 & $02-1047$ & 051854.91 & -693635.8 \\
ELHC 14 & $03-14$ & 051954.90 & -694204.0 \\
ELHC 15 & $07-1108$ & 052723.02 & -695215.9 \\
ELHC 16 & $03-689$ & 052028.00 & -693536.5 \\
ELHC 17 & $07-1120$ & 052724.92 & -695150.6 \\
ELHC 18 & $07-1098$ & 052723.20 & -695201.9 \\
ELHC 19 & $08-649$ & 051711.80 & -692554.0 \\
ELHC 20 & $08-842$ & 051622.50 & -692019.3 \\
ELHC 21 & $10-430$ & 051945.00 & -692616.4 \\
\hline & & & \\
\hline
\end{tabular}

type of variability. A possible explanation involves the inferred lower dust to gas ratio in the LMC due to its lower metallicity, causing less obscuring dust in the vicinity of the ELHCs and making them less variable.

Additional observations are needed to confirm the stars' $\mathrm{HAeBe}$ nature and their properties. For the complete population of ELHCs to be considered LMC PMS stars, emission line spectra and IR excess radiation are tests of cardinal importance.

Acknowledgements. We are grateful to F. Palla for useful discussions. We are also grateful to Romke Bontekoe (Sterrenkundig Instituut Pannekoek, Amsterdam) and Do Kester (SRON, Groningen) for providing us with the new high resolution (Bontekoe et al. 1994) IRAS image of the LMC at 60 micron.

\section{Appendix A: Coordinates, identification and finding charts}

In Table A.1 we present the coordinates and EROS1 identification names of the LMC HAeBe candidates presented in this paper. Figure A.1 presents the finding charts of all the objects. 

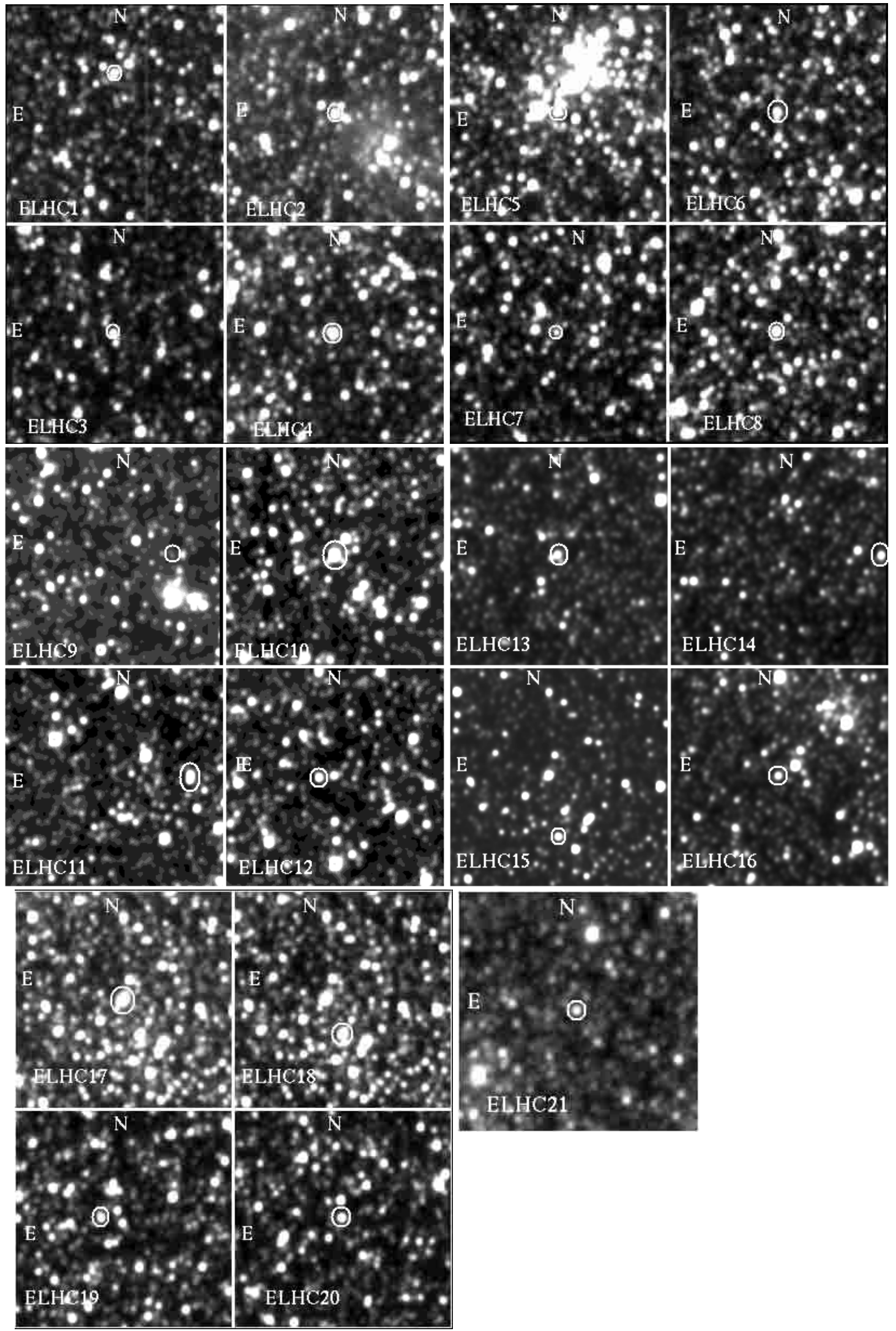

Fig. A.1. Finding chart of the 21 ELHCs in the bar of the Large Magellanic Cloud. Images are taken with the $R_{\mathrm{E}}$ filter. Each panel covers $1.5 \mathrm{x}$ $1.5 \operatorname{arcmin}^{2}$. Orientation and name of the target are indicated on each panel. The target is centred in the circle, not necessarily in the middle of the field. 


\section{References}

Arnaud, M., Aubourg, E., Bareyre, P., et al. 1994a, Experimental Astron., 4, 265

Arnaud, M., Aubourg, E., Bareyre, P., et al. 1994b, Experimental Astron., 4, 279

Beaulieu, J. P., Grison, P., \& Tobin, W. E. 1995, A\&A, 303, 137

Beaulieu, J. P., Krockenberger, M., Sasselov, D. D., et al. 1997a, A\&A, 321, L5

Beaulieu, J. P., Lamers, H. J. G. L. M., Grison, P., et al. 1996, Science, 272,995

Beaulieu, J. P., Sasselov, D. D., Renault, C., et al. 1997b, A\&A, 318, L47

Berrilli, F., Corciulo, G., Ingrosso, G., et al. 1992, ApJ, 398, 254

Bibo, E. A., \& Thé, P. S. 1991, A\&AS, 89, 319

Bontekoe, T. R., Koper, E., Kester, D. J. M. 1994, A\&A, 284, 1037

Catala, C., Donati, J. F., Böhm, T., et al. 1999, A\&A, 345, 884

Draine, B. T. 1985, ApJS, 57, 587

Finkenzeller, U., \& Mundt, R. 1984, A\&AS, 55, 109

Fitzpatrick, E. L. 1986, AJ, 92, 1068

Grinin, V. P. 1988, Soviet Astron. Lett., 14, 27

Grison, P. 1994, A\&A, 289, 404

Grison, P., Beaulieu, J. P., Pritchard, J. D., et al. 1995, A\&AS, 109, 447

Herbig, G. H. 1960, ApJS, 4, 337

Herbst, W., Herbst, D. K., \& Grossman, E. J. 1994, AJ, 108, 1906

Herbst, W., \& Shevchenko, V. S. 1999, AJ, 118, 1043

Horne, J. H., \& Baliunas, S. L. 1986, ApJ, 302, 757

Lamers, H. J. G. L. M., Beaulieu, J. P., \& De Wit, W. J. 1999, A\&A, 341,827

Laval, A., Rosado, M., Boulesteix, J., et al. 1992, A\&A, 253, 213

Mathis, J. S., Rumpl, W., \& Nordsieck, K. H. 1977, ApJ, 217, 425

Mead, K. N., Kutner, M. L., \& Evans, N. J. I. 1990, ApJ, 354, 492
Natta, A., \& Whitney, B. A. 2000, A\&A, 364, 633

Palla, F., \& Stahler, S. W. 1993, ApJ, 418, 414

Palla, F., \& Stahler, S. W. 1994, in The nature and evolutionary status of Herbig Ae/Be stars, ed. Pik Sin Thé, Mario, R. Perez, \& Edward P. J. Van den Heuvel, ASP Conf. Ser., 62, 391

Rostopchina, A. N., Grinin, V. P., Okazaki, A., et al. 1997, A\&A, 327, 145

Sasselov, D. D., Beaulieu, J. P., Renault, C., et al. 1997, A\&A, 324, 471

Scargle, J. D. 1982, ApJ, 263, 835

Schmidt-Kaler, T. 1982, in Landolt Bornstein Vol. 2, Subvol B (Spinger Verlag, Berlin)

Schwarzenberg-Czerny, A. 1989, MNRAS, 241, 153

Schwarzenberg-Czerny, A. 1998, MNRAS, 301, 831

Testi, L., Palla, F., \& Natta, A. 1998, A\&AS, 133, 81

Thé, P. S. 1994, in The nature and evolutionary status of Herbig Ae/Be stars, ed. Pik Sin Thé, Mario R. Perez, \& Edward P. J. Van den Heuvel, ASP Conf. Ser., 62., 23

Van den Ancker, M. E., De Winter, D., \& Tjin A Djie, H. R. E. 1998, A\&A, 330, 145

Voshchinnikov, N. V., \& Grinin, V. P. 1991, Astrofizika, 34, 181

Voshchinnikov, N. V., Grinin, V. P., \& Karjukin, V. V. 1995, A\&A, 294, 547

Voshchinnikov, N. V., Molster, F. J., \& Thé, P. S. 1996, A\&A, 312 243

Walker, A. 1999, in Post-Hipparcos cosmic candles, Astrophysics and space science library, Vol. 237., ed. A. Heck, \& F. Caputo., 125

Waters, L. B. F. M., \& Waelkens, C. 1998, ARA\&A, 36, 233

Wenzel, W. 1969, in Non-periodic Phenomena in Variable Stars, IVth Coll. on Variable Stars, Budapest, p. 61

Wolfire, M. G., \& Cassinelli, J. P. 1986, ApJ, 310, 207

Zaitseva, G. V. 1987, Astrophysics, 25, 626 